\title{
LMI conditions for some dynamical behaviors of fractional-order quaternion-valued neural networks
}

\author{
Dongyuan Lin', Xiaofeng Chen ${ }^{1,2^{*}}$ (D), Bing Li and Xujun Yang
}

\author{
"Correspondence: xxffch@126.com \\ 1 Department of Mathematics, \\ Chongqing Jiaotong University, \\ Chongqing, China \\ 2Department of Economics and \\ Management, Chongqing Jiaotong \\ University, Chongqing, China
}

\begin{abstract}
This paper addresses the issue of three dynamical behaviors including global Mittag-Leffler stability, robust stability and projection synchronization for fractional-order quaternion-valued neural networks (FQVNNs). Some linear matrix inequality conditions for these dynamical behaviors of FQVNNs are given by Lyapunov stability theory, quaternion matrix theory, Homeomorphic mapping theory and fractional differential equation theory. Furthermore, these obtained sufficient conditions for stability and synchronization are superior to those in existing literature. Finally, three examples are given to illustrate the effectiveness of the theoretical results.

Keywords: Fractional-order quaternion-valued neural networks; Global Mittag-Leffler stability; Projective synchronization; Robust stability; Linear matrix inequality
\end{abstract}

\section{Introduction}

Fractional calculus, as a prolongation of integer calculus, was traceable in the 17th century $[1,2]$. In the past, fractional calculus have not been paid enough attention due to the lack of proper application background. In recent decades, various dynamic systems in many areas, such as fluid mechanics, diffusion and wave propagation, boundary layer effects and electromagnetic waves, can be modeled by fractional-order equations, which are a wonderful means to describe the memory and genetic properties of sundry materials and processes compared with integer-order ones [3-10]. On account of this, the research of fractional calculus has fascinated the interest of many scholars in science and engineering $[11,12]$. In recent years, fractional derivatives have been brought into neural networks, in which fractional-order equations can describe their behaviors [13-15]. Thereafter, the dynamics of fractional-order neural networks (FNNs) has been a topic of attention in control and system engineering.

It is worth mentioning that the qualitative analysis of the dynamical behaviors is a critical step for the designed FNNs in many real applications. Generally, the design value of the system parameters deviates from its actual value because of the influence caused by the inaccurate measurement and environmental factors. Accordingly, it is necessary to research the robust stability problem of the system. This problem requires us to set some appropri-

(c) The Author(s) 2019. This article is distributed under the terms of the Creative Commons Attribution 4.0 International License (http://creativecommons.org/licenses/by/4.0/), which permits unrestricted use, distribution, and reproduction in any medium, provided you give appropriate credit to the original author(s) and the source, provide a link to the Creative Commons license, and indicate if changes were made. 
ate intervals so that the system is always stable provided the parameters lie in these intervals. Furthermore, for drive-response FNNs, it is critical to design some kinds of control laws in order to ensure the synchronization between the drive and response ones. Recently, a great deal of outstanding results about the stability, the robust stability and synchronization of FNNs were obtained [16-29]. In [16], $\alpha$-stability and $\alpha$-synchronization were investigated for FNNs. The finite-time stability is was investigated for FNNs in [17]. The robust stability problems for discrete-time uncertain neural networks were discussed in [19]. The Mittag-Leffler stability and synchronization of FNNs with leakage time-varying delay were concerned in [20,21], and the projective synchronization of FNNs was studied in $[22,23]$.

It should be noted that quaternion, discovered by British mathematician W.R. Hamilton in 1843 [30], provides a concise mathematical method to represent automorphisms in three-dimensional and four-dimensional spaces. Compared with matrix representation, the quaternion one is more compact and the calculating speed is faster [31]. Therefore, quaternion-based applications are increasingly emerging in quantum mechanics, signal processing, computer graphics, orbital mechanics and other fields [32, 33]. Particularly, quaternion-valued neural networks (QVNNs), as a universal promotion of real-valued neural networks (RVNNs) and complex-valued neural networks (CVNNs), have been designed for digital image associative memories in [34]. In this application, the three imaginary parts $l, J$ and $\kappa$ of the designed QVNNs are employed to represent the three basic colors separately. In this way, the dimension of the system is greatly reduced and the computational efficiency is greatly improved. In recent years, many researchers have investigated multifarious dynamical behaviors of QVNNs [35-43]. The global $\mu$-stability criteria for QVNNs with unbounded time-varying delays were established in [35, 36, 39]. The robust stability problem of QVNNs with time delays and parameter uncertainties was studied in [40]. The global exponential stability for QVNNs with time-varying delays was researched in [41]. In [42], the authors considered the dissipativity of QVNNs and obtained some succinct criteria for ensuring the QVNNs to be globally dissipative. In [43], a stability analysis was made for continuous-time and discrete-time QVNNs with linear threshold neurons.

To the best of our knowledge, the existing literature on dynamical behaviors of fractional-order QVNNs (FQVNNs) is very little [44]. Motivated by the above discussions, this paper will mainly focus on the global Mittag-Leffler stability, robust stability and projection synchronization of FQVNNs. The main contributions made in this paper are as follows:

(1) Different from the approaches in the existing literature, we investigate the dynamical behaviors of FQVNNs directly instead of converting them into complex-valued or real-valued system, which avoids the increase of system dimension.

(2) Inspired by the product rule for integer-order derivatives, the fractional-order version of the rule is formulated by an inequality, which plays an important role in the computation of the fractional-order derivative of Lyapunov functions in Lyapunov's second method for analyzing dynamical behaviors of fractional-order system.

(3) The quaternion-valued linear matrix inequality (LMI) conditions for the dynamical behaviors of FQVNNs are converted into the complex-valued LMI ones, which can be tested directly by the mathematical software MATLAB. 
Notation: In this paper, $\mathbb{R}, \mathbb{C}$ and $\mathbb{H}$ denote separately the real field, the complex field and the skew field of a quaternion. $\mathbb{R}^{n \times m}, \mathbb{C}^{n \times m}$ and $\mathbb{H}^{n \times m}$, simply, $\mathbb{R}^{n}, \mathbb{C}^{n}$, and $\mathbb{H}^{n}$ when $m=1$, denote separately $n \times m$ matrices with entries from $\mathbb{R}, \mathbb{C}$ and $\mathbb{H}$. The standard imaginary units in $\mathbb{H}$ are denoted by $l, J$ and $\kappa$ which satisfy $\iota^{2}=J^{2}=\kappa^{2}=-1, l J=-\jmath \imath=$ $\kappa, \jmath \kappa=-\kappa \jmath=\imath, \kappa l=-\imath \kappa=\jmath$. For a quaternion $q=q_{0}+q_{1} \imath+q_{2} J+q_{3} \kappa=\left(q_{0}+q_{1} l\right)+$ $\left(q_{2}+q_{3} l\right) J \in \mathbb{H}$, where $q_{0}, q_{1}, q_{2}, q_{3} \in \mathbb{R}$. Let $q^{*}=q_{0}-q_{1} l-q_{2} J-q_{3} \kappa$ be the conjugate of $q$, and $|q|=\sqrt{q_{0}^{2}+q_{1}^{2}+q_{2}^{2}+q_{3}^{2}}$ be the modulus of $q$. For $z=\left(z_{1}, z_{2}, \ldots, z_{n}\right)^{T} \in \mathbb{H}^{n}$, let $|z|=\left(\left|z_{1}\right|,\left|z_{2}\right|, \ldots,\left|z_{n}\right|\right)^{T}$ be the modulus of $z$, and $\|z\|=\sqrt{\sum_{i=1}^{n}\left|z_{i}\right|^{2}}$ be the norm of $z$. In addition, let $h^{(11)}, h^{(12)}, h^{(21)}, h^{(22)} \in \mathbb{R}$ denote separately the first, second, third and fourth parts of $h$, that is, $h^{(11)}=q_{0}, h^{(12)}=q_{1}, h^{(21)}=q_{2}$ and $h^{(22)}=q_{3}$, and let $h_{1}, h_{2} \in \mathbb{C}$ denote separately the first and second complex parts of $h$, that is, $h_{1}=q_{0}+q_{1} l$ and $h_{2}=q_{2}+q_{3} l$. In the same way, for $A \in \mathbb{H}^{n \times m}$, the first and second complex parts are denoted by $A_{1}$ and $A_{2}$, and we have $A=A_{1}+A_{2} l$. The notation $\bar{A}, A^{T}$ and $A^{*}$ stand for the conjugate, the transpose and the conjugate transpose, separately, of the matrix $A$. For $A=\left(a_{i j}\right)_{n \times n} \in \mathbb{H}^{n \times n}$, let $\|A\|=$ $\sqrt{\sum_{i=1}^{n} \sum_{j=1}^{n}\left|a_{i j}\right|^{2}}$ denote the norm of $A$. I denotes the identity matrix with appropriate dimensions. The notation $X \geq Y$ (separately, $X>Y$ ) means that $X-Y$ is positive semidefinite (separately, positive definite). For a positive definite Hermitian matrix $P \in \mathbb{H}^{n \times n}$, $\lambda_{\max }(P)$ and $\lambda_{\min }(P)$ are defined as the largest and the smallest eigenvalues of $P$, separately.

\section{Model description and preliminaries}

In order to describe the model considered in this paper, we first introduce the definition of the fractional derivative.

Definition 1 ([22]) The Caputo fractional derivative of order $\alpha$ for a function $f(t) \in$ $C^{n+1}([0,+\infty), \mathbb{H})$ is defined as

$$
D^{\alpha} f(t)=\frac{1}{\Gamma(n-\alpha)} \int_{0}^{t} \frac{f^{n}(\tau)}{(t-\tau)^{\alpha-n+1}} d \tau
$$

where $\alpha>0, \Gamma(\cdot)$ represents the gamma function and $n$ is a positive integer such that $n-1<\alpha<n$. Particularly, when $0<\alpha<1$,

$$
D^{\alpha} f(t)=\frac{1}{\Gamma(1-\alpha)} \int_{0}^{t} \frac{f^{\prime}(\tau)}{(t-\tau)^{\alpha}} d \tau
$$

The Laplace transform of the Caputo fractional-order derivative is

$$
\mathcal{L}\left\{D^{\alpha} f(t) ; s\right\}=s^{\alpha} F(s)-\sum_{k-1}^{n-1} s^{\alpha-k-1} f^{(k)}\left(t_{0}\right),
$$

where $n-1<\alpha \leq n, \mathcal{L}\{\cdot\}$ is the Laplace transform, $s$ is the variable in the Laplace domain, and $F(s)=\mathcal{L}\{f(t)\}$.

We consider the FQVNNs model with the following form:

$$
D^{\alpha} x(t)=-C x(t)+B f(x(t))+I
$$

where $\alpha \in(0,1), t \geq 0, x(t)=\left(x_{1}(t), x_{2}(t), \ldots, x_{n}(t)\right)^{T} \in \mathbb{H}^{n}$ is the state vector of the neural network with $n$ neurons at time $t$; $C=\operatorname{diag}\left\{c_{1}, c_{2}, \ldots, c_{n}\right\} \in \mathbb{R}^{n \times n}$ is the self-feedback 
connection weight matrix; $B \in \mathbb{H}^{n \times n}$ is the interconnection weight matrix; $f(x(t))=$ $\left(f_{1}(x(t)), f_{2}(x(t)), \ldots, f_{n}(x(t))\right)^{T} \in \mathbb{H}^{n}$ denotes the neuron activation at time $t$, and $I \in \mathbb{H}^{n}$ denotes the external input vector.

The initial condition associated with system (2) is of the form

$$
x(0)=x_{0},
$$

where $x_{0} \in \mathbb{H}^{n}$.

In order to study the dynamical behaviors of system (2), the Lipschitz condition for the activation function is usually needed. So we assume that

Assumption 1 For $i=1,2, \ldots, n, f_{i}(x)$ is continuous and satisfies

$$
\left|f_{i}(y)-f_{i}(x)\right| \leq l_{i}|y-x|, \quad \forall x, y \in \mathbb{H}
$$

where $l_{i}$ is a real constant. Moreover, define $L=\operatorname{diag}\left\{l_{1}, l_{2}, \ldots, l_{n}\right\}$.

In addition, the following assumption on parameter ranges is needed to investigate the robust stability of system (2).

Assumption 2 The parameters $C, B, I$ in FQVNNs (2) are assumed to be in the following sets, respectively:

$$
\begin{aligned}
& C_{I}=[\check{C}, \hat{C}]=\left\{C \in \mathbb{H}^{n \times n}: \check{C} \preceq C \preceq \hat{C}\right\}, \\
& B_{I}=[\check{B}, \hat{B}]=\left\{B \in \mathbb{H}^{n \times n}: \check{B} \preceq B \preceq \hat{B}\right\}, \\
& I_{I}=[\check{I}, \hat{I}]=\left\{I \in \mathbb{H}^{n}: \check{I} \preceq I \preceq \hat{I}\right\},
\end{aligned}
$$

where $\check{C}, \hat{C}, \check{B}, \hat{B} \in \mathbb{H}^{n \times n}$ and $\check{I}, \hat{I} \in \mathbb{H}^{n}$. Furthermore, let $\check{C}=\left(\check{c}_{i j}\right)_{n \times n}, \hat{C}=\left(\hat{c}_{i j}\right)_{n \times n}, \check{B}=$ $\left(\check{b}_{i j}\right)_{n \times n}, \hat{B}=\left(\hat{b}_{i j}\right)_{n \times n}$. Then we define $\tilde{C}=\left(\tilde{c}_{i j}\right)_{n \times n}, \tilde{B}=\left(\tilde{b}_{i j}\right)_{n \times n}$, where $\tilde{c}_{i j}=\max \left\{\left|\check{c}_{i j}\right|,\left|\hat{c}_{i j}\right|\right\}$, $\tilde{b}_{i j}=\max \left\{\left|\check{b}_{i j}\right|,\left|\hat{b}_{i j}\right|\right\}$.

In order to discuss the synchronization problem between two FQVNNs, we introduce the response system associated with the drive system (2) as follows:

$$
D^{\alpha} y(t)=-C y(t)+B f(y(t))+I+u(t)
$$

where $y(t) \in \mathbb{H}^{n}$ is the state vectors, $u(t) \in \mathbb{H}^{n}$ is a suitable control law to be designed, and other parameters are the same as (2).

Next we introduce some definitions and lemmas about fractional-order system and quaternion matrix.

Definition 2 ([22]) With two parameters $\alpha>0$ and $\beta>0$, the Mittag-Leffler function is defined as

$$
E_{\alpha, \beta}(z)=\sum_{k=0}^{\infty} \frac{z^{k}}{\Gamma(\alpha k+\beta)} .
$$


When $\beta=1$, its one-parameter form can be rewritten as

$$
E_{\alpha}(z)=\sum_{k=0}^{\infty} \frac{z^{k}}{\Gamma(k \alpha+1)}
$$

where $z \in \mathbb{H}$. Particularly, $E_{1}(z)=e^{z}$.

Definition 3 The constant $\tilde{x} \in \mathbb{H}^{n}$ is an equilibrium point of FQVNNs (2) if and only if

$$
-C \tilde{x}+B f(\tilde{x})+I=0 .
$$

Definition 4 An equilibrium point $\tilde{x}$ of FQVNNs (2) is said to be Mittag-Leffler stable if there exist two positive constants $\lambda$ and $\mu$, such that, for any solution $x(t)$ of FQVNNs (2) with the initial condition (3), one has

$$
\|x(t)-\tilde{x}\| \leq\left\{m\left(x_{0}-\tilde{x}\right) E_{\alpha}\left(-\lambda t^{\alpha}\right)\right\}^{\mu}, \quad t \geq 0
$$

where $m(\cdot)$ is a locally Lipschitz function on $\mathbb{D} \subseteq \mathbb{H}^{n}$ satisfying $m(0)=0$ and $m(x) \geq 0$.

Definition 5 FQVNNs (2) with the parameters ranges defined by Assumption 2 are globally Mittag-Leffler robust stable if the unique equilibrium point of (2) is Mittag-Leffler robust stable for all $C \in C_{I}, B \in B_{I}$ and $I \in I_{I}$.

Definition 6 Systems (2) and (4) are globally Mittag-Leffler projective synchronized, if there exists a projective coefficient matrix $\Upsilon \in \mathbb{H}^{n \times n}$, two positive constants $\lambda$ and $\mu$ such that, for any two solutions $x(t)$ and $y(t)$ of system (2) and system (4) with different initial values denoted by $x_{0}, y_{0} \in \mathbb{H}^{n}$, one has

$$
\|y(t)-\Upsilon x(t)\| \leq\left\{m\left(y_{0}-\Upsilon x_{0}\right) E_{\alpha}\left(-\lambda t^{\alpha}\right)\right\}^{\mu}, \quad t \geq 0,
$$

where $m(\cdot)$ is a locally Lipschitz function on $\mathbb{D} \subseteq \mathbb{H}^{n}$ satisfying $m(0)=0$ and $m(x) \geq 0$.

Lemma 1 ([45]) Let $A=A_{1}+A_{2} J$ and $B=B_{1}+B_{2} J$, where $A_{1}, A_{2}, B_{1}, B_{2} \in \mathbb{C}^{n \times n}$ and $A, B \in$ $\mathbb{H}^{n \times n}$. Then:

(1) $A^{*}=A_{1}^{*}-A_{2}^{T} J$;

(2) $A B=\left(A_{1} B_{1}-A_{2} \bar{B}_{2}\right)+\left(A_{1} B_{2}+A_{2} \bar{B}_{1}\right) J$, where $\bar{B}_{1}$ and $\bar{B}_{2}$ are the conjugate of $B_{1}$ and $B_{2}$, respectively.

Lemma 2 ([45]) Let $Q \in \mathbb{H}^{n \times n}$ be a Hermitian matrix and $Q=Q_{1}+Q_{2} J$, where $Q_{1}, Q_{2} \in$ $\mathbb{C}^{n \times n}$. Then $Q<0$ is equivalent to

$$
\left(\begin{array}{cc}
Q_{1} & -Q_{2} \\
\bar{Q}_{2} & \bar{Q}_{1}
\end{array}\right)<0
$$

where $\bar{Q}_{1}, \bar{Q}_{2}$ are the conjugate matrices of $Q_{1}$ and $Q_{2}$, respectively. 
Lemma 3 ([40]) For any $a, b \in \mathbb{H}^{n}$, if $P \in \mathbb{H}^{n \times n}$ is a positive definite Hermitian matrix, then $a^{*} b+b^{*} a \leq a^{*} P a+b^{*} P^{-1} b$.

Lemma 4 ([40]) A given real symmetric matrix

$$
S=\left(\begin{array}{ll}
S_{11} & S_{12} \\
S_{21} & S_{22}
\end{array}\right)<0,
$$

where $S_{11}^{T}=S_{11}, S_{12}^{T}=S_{21}$, and $S_{22}^{T}=S_{22}$, if and only if any of the following two conditions holds:

(i) $S_{22}<0$ and $S_{11}-S_{12} S_{22}^{-1} S_{21}<0$,

(ii) $S_{11}<0$ and $S_{22}-S_{21} S_{11}^{-1} S_{12}<0$.

Lemma 5 ([40]) If $H(x): \mathbb{H}^{n} \rightarrow \mathbb{H}^{n}$ is a continuous map and satisfies the following conditions:

(i) $H(x)$ is injective on $\mathbb{H}^{n}$,

(ii) $\lim _{\|x\| \rightarrow \infty}\|H(x)\|=\infty$.

Then $H(x)$ is a homeomorphism of $\mathbb{H}^{n}$ onto itself.

Lemma 6 Let $\tilde{x}$ is an equilibrium point of FQVNNs (2) with initial condition (3), and $\mathbb{D} \subseteq$ $\mathbb{H}^{n}$ be a domain containing the origin. Let $V(x): \mathbb{D} \rightarrow \mathbb{R}$ be a continuously differentiable function and locally Lipschitz such that

$$
\begin{aligned}
& a_{1}\|x(t)\|^{a} \leq V(x(t)) \leq a_{2}\|x(t)\|^{a b}, \\
& D^{\alpha} V(x(t)) \leq-a_{3}\|x(t)\|^{a b},
\end{aligned}
$$

where $t \geq 0, \alpha \in(0,1), x(t) \in \mathbb{D}, a_{1}, a_{2}, a_{3}, a$, and $b$ are arbitrary positive constants. Then $\tilde{x}$ is Mittag-Leffler stable.

Proof By (6) and (7), it is obvious that

$$
\begin{aligned}
& a_{1}\|x(t)-\tilde{x}\|^{a} \leq V(x(t)-\tilde{x}) \leq a_{2}\|x(t)-\tilde{x}\|^{a b}, \\
& D^{\alpha} V(x(t)-\tilde{x}) \leq-a_{3}\|x(t)-\tilde{x}\|^{a b} .
\end{aligned}
$$

It follows from (8) and (9) that

$$
D^{\alpha} V(x(t)-\tilde{x}) \leq-\beta V(x(t)-\tilde{x})
$$

where $\beta=a_{2}^{-1} a_{3}$. Then there exists $M(t) \geq 0$ such that

$$
D^{\alpha} V(x(t)-\tilde{x})+M(t)=-\beta V(x(t)-\tilde{x}) .
$$

By the application of Laplace transform to (10), we obtain

$$
s^{\alpha} \mathcal{V}(s)-s^{\alpha-1} V_{0}+\mathcal{M}(s)=-\beta \mathcal{V}(s)
$$


where $V_{0}=V\left(x_{0}-\tilde{x}\right), \mathcal{V}(s)=\mathcal{L}\{V(x(t)-\tilde{x})\}, \mathcal{M}(s)=\mathcal{L}\{M(t)\}, \mathcal{L}\{\cdot\}$ is the Laplace transform. From (11), we could compute

$$
\mathcal{V}(s)=\frac{V_{0} s^{\alpha-1}-\mathcal{M}(s)}{s^{\alpha}+\beta}
$$

On the one hand, when $x_{0}=\tilde{x}$, we see that $x(t)=\tilde{x}$ is the solution of (2) and $V_{0}=0$. On the other hand, when $x_{0} \neq \tilde{x}$, then $V_{0}>0$. Since $V(x)$ is locally Lipschitz, we get

$$
V(x(t)-\tilde{x})=V_{0} E_{\alpha}\left(-\beta t^{\alpha}\right)-M(t) *\left[t^{\alpha-1} E_{\alpha, \alpha}\left(-\beta t^{\alpha}\right)\right]
$$

by the inverse Laplace transform of (12). Noting that $t^{\alpha-1} \geq 0$ and $E_{\alpha, \alpha}\left(-\beta t^{\alpha}\right) \geq 0$ for all $t \in[0,+\infty)$, we have

$$
V(x(t)-\tilde{x}) \leq V_{0} E_{\alpha}\left(-\beta t^{\alpha}\right)
$$

In view of (8), it follows that

$$
\|x(t)-\tilde{x}\| \leq\left\{m\left(x_{0}-\tilde{x}\right) E_{\alpha}\left(-\beta t^{\alpha}\right)\right\}^{\frac{1}{a}}
$$

where $m\left(x_{0}-\tilde{x}\right)=a_{1}^{-1} V_{0}=a_{1}^{-1} V\left(x_{0}-\tilde{x}\right)$. Obviously, $m\left(x_{0}-\tilde{x}\right) \geq 0$ and $m\left(x_{0}-\tilde{x}\right)=0$ if and only if $x_{0}-\tilde{x}=0$. Moreover, $m(\cdot)$ is locally Lipschitz because $V(\cdot)$ is locally Lipschitz. Therefore, the equilibrium point $\tilde{x}$ of FQVNNs (2) is Mittag-Leffler stable by Definition 4.

Lemma 7 Let $x(t)=\left(x_{1}(t), x_{2}(t), \ldots, x_{n}(t)\right)^{T} \in \mathbb{H}^{n}$, where $x_{i}(t), i=1,2, \ldots, n$, are continuous and differentiable function, and $P \in \mathbb{H}^{n \times n}$ be a positive definite Hermitian matrix. Then, for $t \geq 0$, the following inequality holds:

$$
D^{\alpha}\left(x^{*}(t) P x(t)\right) \leq\left[D^{\alpha} x(t)\right]^{*} P x(t)+x^{*}(t) P D^{\alpha} x(t) .
$$

Proof By Definition 1, we see that

$$
\begin{aligned}
{\left[D^{\alpha} x(t)\right]^{*} P x(t)+x^{*}(t) P D^{\alpha} x(t)-D^{\alpha}\left(x^{*}(t) P x(t)\right) } \\
=\left[\frac{1}{\Gamma(1-\alpha)} \int_{0}^{t} \frac{x^{\prime}(\tau)}{(t-\tau)^{\alpha}} d \tau\right]^{*} P x(t) \\
\quad+x^{*}(t) P\left[\frac{1}{\Gamma(1-\alpha)} \int_{0}^{t} \frac{x^{\prime}(\tau)}{(t-\tau)^{\alpha}} d \tau\right] \\
\quad-\frac{1}{\Gamma(1-\alpha)} \int_{0}^{t} \frac{\left[x^{*}(\tau) P x(\tau)\right]^{\prime}}{(t-\tau)^{\alpha}} d \tau \\
=\frac{1}{\Gamma(1-\alpha)} \int_{0}^{t} \frac{\left[x^{*}(\tau)\right]^{\prime} P[x(t)-x(\tau)]}{(t-\tau)^{\alpha}} d \tau \\
\quad+\frac{1}{\Gamma(1-\alpha)} \int_{0}^{t} \frac{\left[x^{*}(t)-x^{*}(\tau)\right] P x^{\prime}(\tau)}{(t-\tau)^{\alpha}} d \tau .
\end{aligned}
$$


Let $y(\tau)=x(t)-x(\tau)$. Then (14) can be written as

$$
\begin{aligned}
& \left(D^{\alpha} x(t)\right)^{*} P x(t)+x^{*}(t) P D^{\alpha} x(t)-D^{\alpha}\left(x^{*}(t) P x(t)\right) \\
& \quad=-\frac{1}{\Gamma(1-\alpha)} \int_{0}^{t} \frac{\left[y^{*}(\tau)\right]^{\prime} P y(\tau)+y^{*}(\tau) P y^{\prime}(\tau)}{(t-\tau)^{\alpha}} d \tau \\
& =-\frac{1}{\Gamma(1-\alpha)} \int_{0}^{t} \frac{d\left(y^{*}(\tau) P y(\tau)\right)}{(t-\tau)^{\alpha}} \\
& =\frac{1}{\Gamma(1-\alpha)}\left[\int_{0}^{t} y^{*}(\tau) P y(\tau) d\left(\frac{1}{(t-\tau)^{\alpha}}\right)-\left.\frac{y^{*}(\tau) P y(\tau)}{(t-\tau)^{\alpha}}\right|_{0} ^{t}\right] \\
& =\frac{1}{\Gamma(1-\alpha)}\left[-\left.\frac{y^{*}(\tau) P y(\tau)}{(t-\tau)^{\alpha}}\right|_{\tau=t}+\frac{y^{*}(0) P y(0)}{(t-0)^{\alpha}}+\int_{0}^{t} \frac{\alpha y^{*}(\tau) P y(\tau)}{(t-\tau)^{\alpha+1}} d \tau\right] .
\end{aligned}
$$

On the other hand, by using Lopida's law, we could compute

$$
\begin{aligned}
\left.\frac{y^{*}(\tau) P y(\tau)}{(t-\tau)^{\alpha}}\right|_{\tau=t} & =\lim _{\tau \rightarrow t} \frac{y^{*}(\tau) P y(\tau)}{(t-\tau)^{\alpha}} \\
& =\lim _{\tau \rightarrow t} \frac{[x(t)-x(\tau)]^{*} P[x(t)-x(\tau)]}{(t-\tau)^{\alpha}} \\
& =\lim _{\tau \rightarrow t} \frac{(t-\tau)^{1-\alpha}}{-\alpha}\left[\left(x^{*}(\tau)-x^{*}(t)\right) P x^{\prime}(\tau)+\left[x^{*}(\tau)\right]^{\prime} P(x(\tau)-x(t))\right] \\
& =0 .
\end{aligned}
$$

Substituting (16) into (15), we obtain

$$
\begin{aligned}
& {\left[D^{\alpha} x(t)\right]^{*} P x(t)+x^{*}(t) P D^{\alpha} x(t)-D^{\alpha}\left(x^{*}(t) P x(t)\right)} \\
& \quad=\frac{1}{\Gamma(1-\alpha)}\left[\frac{y^{*}(0) P y(0)}{(t-0)^{\alpha}}+\int_{0}^{t} \frac{y^{*}(\tau) P y(\tau)}{\alpha(t-\tau)^{\alpha-1}} d \tau\right] \geq 0,
\end{aligned}
$$

since $P$ is a positive definite Hermitian matrix.

Lemma 8 ([40]) Suppose $A \in \mathbb{H}^{n \times n}, \check{A}=\left(\check{a}_{i j}\right)_{n \times n} \in \mathbb{H}^{n \times n}, \hat{A}=\left(\hat{a}_{i j}\right)_{n \times n} \in \mathbb{H}^{n \times n}$, and $\check{A} \preceq A \preceq$ $\hat{A}$. Then, for all $x, y \in \mathbb{H}^{n}$, the following inequalities hold:

$$
\begin{aligned}
& x^{*} A^{*} A x \leq|x|^{*}|A|^{*}|A||x| \leq|x|^{*} \tilde{A}^{*} \tilde{A}|x|, \\
& x^{*} A^{*} y+y^{*} A x \leq 2|x|^{*}|A|^{*}|y| \leq 2|x|^{*} \tilde{A}^{*}|y|,
\end{aligned}
$$

where $\tilde{A}=\left(\tilde{a}_{i j}\right)_{n \times n}, \tilde{a}_{i j}=\max \left\{\left|\check{a}_{i j}\right|,\left|\hat{a}_{i j}\right|\right\}$.

Remark 1 In Definition 6, we say system (2) and system (4) are globally Mittag-Leffler complete projective synchronized if $\Upsilon=E$, and they are globally Mittag-Leffler projective anti-synchronized if $\Upsilon=-E$.

Remark 2 The class of the activation functions $f(x(t)) \in \mathbb{H}^{n}$ satisfying Assumption 1 includes the linear threshold function defined in [44]. 
Remark 3 Differen from the product rule for integer-order derivatives, the fractionalorder version of the rule in Lemma 7 is presented by an inequality, which will greatly reduce the complexity of calculating the fractional-order derivative of the Lyapunov functions in the proofs for the following main theorems.

\section{Main results}

In this section, we will discuss the existence, uniqueness, global Mittag-Leffler stability and global Mittag-Leffler robust stability of the equilibrium point FQVNNs (2) as well as the synchronization of FQVNNs (2).

Theorem 1 Under Assumption 1, FQVNNs (2) have a unique equilibrium point $\tilde{x}$ which is globally Mittag-Leffler stable, if there exist a real positive diagonal matrix $Q$ and a Hermitian matrix $P>0$ satisfying

$$
\left(\begin{array}{cc}
-C^{*} P-P C+L Q L & P B \\
B^{*} P & -Q
\end{array}\right)<0 .
$$

Proof First, we prove that system (2) has the unique equilibrium point by the homeomorphic mapping theory. Define a mapping $\Theta(\omega): \mathbb{H}^{n} \rightarrow \mathbb{H}^{n}$ and $\Theta(\omega)=-C \omega+B f(\omega)+I$.

On the one hand, we prove the mapping $\Theta(\omega)$ is injective on $\mathbb{H}^{n}$. If there exist $\omega, \tilde{\omega} \in H^{n}$ with $\omega \neq \tilde{\omega}$ such that $\Theta(\omega)=\Theta(\tilde{\omega})$. By Assumption 1, Lemma 3 and Lemma 8, we can compute

$$
\begin{aligned}
0= & (\omega-\tilde{\omega})^{*} P[\Theta(\omega)-\Theta(\tilde{\omega})]+[\Theta(\omega)-\Theta(\tilde{\omega})]^{*} P(\omega-\tilde{\omega}) \\
= & -(\omega-\tilde{\omega})^{*}\left(P C+C^{*} P\right)(\omega-\tilde{\omega})+(\omega-\tilde{\omega})^{*} P B[f(\omega)-f(\tilde{\omega})] \\
& +[f(\omega)-f(\tilde{\omega})]^{*} B^{*} P(\omega-\tilde{\omega}) \\
\leq & (\omega-\tilde{\omega})^{*}\left(-C^{*} P-P C\right)(\omega-\tilde{\omega})+(\omega-\tilde{\omega})^{*} P B Q^{-1} B^{*} P(\omega-\tilde{\omega}) \\
& +[f(\omega)-f(\tilde{\omega})]^{*} Q[f(\omega)-f(\tilde{\omega})] \\
\leq & (\omega-\tilde{\omega})^{*}\left(-C^{*} P-P C\right)(\omega-\tilde{\omega})+(\omega-\tilde{\omega})^{*} P B Q^{-1} B^{*} P(\omega-\tilde{\omega}) \\
& +(\omega-\tilde{\omega})^{*} L Q L(\omega-\tilde{\omega}) \\
\leq & |\omega-\tilde{\omega}|^{*}\left(-C^{*} P-P C+P B Q^{-1} B^{*} P+L Q L\right)|\omega-\tilde{\omega}| .
\end{aligned}
$$

From Lemma 4 and the LMI condition (17) we obtain $-C^{*} P-P C+P B Q^{-1} B^{*} P+L Q L<0$, which means that $\omega=\tilde{\omega}$. Then $\Theta(\omega)$ is an injective mapping.

On the other hand, we need to prove $\|\tilde{\Theta}(\omega)\| \rightarrow \infty$ as $\|\omega\| \rightarrow \infty$. Let $\tilde{\Theta}(\omega)=\Theta(\omega)-$ $\Theta(0)$. According to Assumption 1, Lemma 3 and Lemma 8, we obtain

$$
\begin{array}{rl}
\omega^{*} & P \tilde{\Theta}(\omega)+(\tilde{\Theta}(\omega))^{*} P \omega \\
& =\omega^{*}\left(-C^{*} P-P C\right) \omega+\omega^{*} P B[f(\omega)-f(0)]+[f(\omega)-f(0)]^{*} B^{*} P \omega \\
& \leq \omega^{*}\left(-C^{*} P-P C\right) \omega+\omega^{*} P B Q^{-1} B^{*} P \omega+[f(\omega)-f(0)]^{*} Q[f(\omega)-f(0)] \\
& \leq \omega^{*}\left(-C^{*} P-P C+P B Q^{-1} B^{*} P+L Q L\right) \omega
\end{array}
$$




$$
\begin{aligned}
& \leq|\omega|^{*}\left(-C^{*} P-P C+P B Q^{-1} B^{*} P+L Q L\right)|\omega| \\
& \leq-\lambda_{\min }\left(C^{*} P+P C-P B Q^{-1} B^{*} P-L Q L\right)\|\omega\|^{2} .
\end{aligned}
$$

It follows from the Cauchy-Schwarz inequality that

$$
\lambda_{\min }\left(C^{*} P+P C-P B Q^{-1} B^{*} P-L Q L\right)\|\omega\|^{2} \leq 2\left\|\omega^{*}\right\|\|P\|\|\tilde{\Theta}(\omega)\| .
$$

When $\omega \neq 0$, we have

$$
\|\tilde{\Theta}(\omega)\| \geq \frac{\lambda_{\min }\left(C^{*} P+P C-P B Q^{-1} B^{*} P-L Q L\right)\|\omega\|^{2}}{2\|P\|} .
$$

Therefore, $\|\tilde{\Theta}(\omega)\| \rightarrow \infty$ as $\|\omega\| \rightarrow \infty$. From above two aspects, we could find that $\Theta(\omega)$ is a homeomorphism on $\mathbb{H}^{n}$ by Lemma 5 . Hence, system (2) has a unique equilibrium point.

Then we prove the equilibrium point $\tilde{x}$ of system (2) is globally Mittag-Leffler stable.

With the variable substitution $y(t)=x(t)-\tilde{x}$, we could rewrite system (2) as

$$
\begin{aligned}
D^{\alpha} y(t) & =-C(y(t)+\tilde{x})+B f(y(t)+\tilde{x})+I \\
& =-C y(t)+B[f(y(t)+\tilde{x})-f(\tilde{x})]-C \tilde{x}+B f(\tilde{x})+I \\
& =-C y(t)+B[f(y(t)+\tilde{x})-f(\tilde{x})] \\
& =-C y(t)+B g(y(t)),
\end{aligned}
$$

where $g(y(t))=f(y(t)+\tilde{x})-f(\tilde{x}), g(0)=0$, and for all $u, v \in \mathbb{H}, g(x)$ satisfies

$$
\left|g_{i}(u)-g_{i}(v)\right| \leq l_{i}|u-v|
$$

By doing this variable substitution, system (2) equals the system

$$
D^{\alpha} y(t)=-C y(t)+B g(y(t))
$$

It is obvious that $\tilde{y}=0$ is the equilibrium point of system (20). Consider a Lyapunov function as follows:

$$
V(y(t))=y^{*} P y .
$$

It follows from (21) that

$$
\lambda_{\min }(P)\|y\|_{2}^{2} \leq V(y(t)) \leq \lambda_{\max }(P)\|y\|_{2}^{2} .
$$

Thus, the condition (6) in Lemma 6 is satisfied. Then we do the following calculations by Assumption 1, Lemma 3 and Lemma 7:

$$
\begin{aligned}
& D^{\alpha} V(y(t)) \\
& \quad \leq\left(D^{\alpha} y(t)\right)^{*} P y(t)+[y(t)]^{*} P D^{\alpha} y(t)
\end{aligned}
$$




$$
\begin{aligned}
& =[-C y(t)+B g(y(t))]^{*} P y(t)+[y(t)]^{*} P[-C y(t)+B g(y(t))] \\
& =y^{*}\left(-C^{*} P-P C\right) y+g^{*}(y) B^{*} P y+y^{*} P^{*} B g(y) \\
& \leq y^{*}\left(-C^{*} P-P C\right) y+y^{*} P B Q^{-1} B^{*} P y+g^{*}(y) Q g(y) \\
& =y^{*}\left(-C^{*} P-P C\right) y+y^{*} P B Q^{-1} B^{*} P y+(g(y)-g(0))^{*} Q(g(y)-g(0)) \\
& \leq y^{*}\left(-C^{*} P-P C\right) y+y^{*} P B Q^{-1} B^{*} P y+y^{*} L Q L y \\
& =y^{*}\left(-C^{*} P-P C+P B Q^{-1} B^{*} P+L Q L\right) y \\
& \leq \lambda_{\max }\left(-C^{*} P-P C+P B Q^{-1} B^{*} P+L Q L\right)\|y\|_{2}^{2} .
\end{aligned}
$$

Based on Lemma 4 and condition (17), we see that $\lambda_{\max }\left(-C^{*} P-P C+P B Q^{-1} B^{*} P+L Q L\right)<0$. It shows that the condition (7) in Lemma 6 is satisfied. Hence, the equilibrium point $\tilde{y}=0$ of system (20) is globally Mittag-Leffler stable by Lemma 6 . Namely, the equilibrium point $\tilde{x}$ of (2) is globally Mittag-Leffler stable.

Theorem 2 Under Assumptions 1 and 2, FQVNNs (2) have a unique equilibrium point and the equilibrium point is globally Mittag-Leffler robust stable, if there exist a real positive diagonal matrix $Q$ and a Hermitian matrices $P>0$ such that the following LMI holds:

$$
\left(\begin{array}{cc}
-\check{C}^{*} P-P \check{C}+L Q L & P \tilde{B} \\
\tilde{B}^{*} P & -Q
\end{array}\right)<0 .
$$

Proof Similar to the proof of Theorem 1, we define a mapping $\Theta(\omega)=-C \omega+B f(\omega)+I$. First, we prove the mapping is injective. If there exist $\omega, \tilde{\omega} \in H^{n}$ with $\omega \neq \tilde{\omega}$ such that $\Theta(\omega)=\Theta(\tilde{\omega})$. Based on (18) and Lemma 8, we could write

$$
\begin{aligned}
0 & \leq|\omega-\tilde{\omega}|^{*}\left(-C^{*} P-P C+P B Q^{-1} B^{*} P+L Q L\right)|\omega-\tilde{\omega}| \\
& \leq|\omega-\tilde{\omega}|^{*}\left(-\check{C}^{*} P-P \check{C}+P \tilde{B} Q^{-1} \tilde{B}^{*} P+L Q L\right)|\omega-\tilde{\omega}| .
\end{aligned}
$$

From Lemma 4 and the LMI condition (23), we have $-\check{C}^{*} P-P \check{C}+P \tilde{B} Q^{-1} \tilde{B}^{*} P+L Q L<0$, which means $\omega=\tilde{\omega}$. Hence $\Theta(\omega)$ is an injective mapping.

Let $\tilde{\Theta}(\omega)=\Theta(\omega)-\Theta(0)$. According to (19) and Lemma 8 we get

$$
\begin{aligned}
& \omega^{*} P \tilde{\Theta}(\omega)+(\tilde{\Theta}(\omega))^{*} P \omega \\
& \quad \leq|\omega|^{*}\left(-C^{*} P-P C+P B Q^{-1} B^{*} P+L Q L\right)|\omega| \\
& \quad \leq|\omega|^{*}\left(-\check{C}^{*} P-P \check{C}+P \tilde{B} Q^{-1} \tilde{B}^{*} P+L Q L\right)|\omega| \\
& \quad \leq-\lambda_{\min }\left(\check{C}^{*} P+P \check{C}-P \tilde{B} Q^{-1} \tilde{B}^{*} P-L Q L\right)\|\omega\|^{2} .
\end{aligned}
$$

It follows from the Cauchy-Schwarz inequality that

$$
\lambda_{\min }\left(\check{C}^{*} P+P \check{C}-P \tilde{B} Q^{-1} \tilde{B}^{*} P-L Q L\right)\|\omega\|^{2} \leq 2\left\|\omega^{*}\right\|\|P\|\|\tilde{\Theta}(\omega)\| .
$$

When $\omega \neq 0$, we have

$$
\|\tilde{\Theta}(\omega)\| \geq \frac{\lambda_{\min }\left(\check{C}^{*} P+P \check{C}-P \tilde{B} Q^{-1} \tilde{B}^{*} P-L Q L\right)\|\omega\|^{2}}{2\|P\|} .
$$


It is obvious that $\|\tilde{\Theta}(\omega)\| \rightarrow \infty$ as $\|\omega\| \rightarrow \infty$. From Lemma $5, \Theta(\omega)$ is a homeomorphism of $\mathbb{H}^{n}$. Therefore, system (2) has the unique equilibrium point.

With the variable substitution $y(t)=x(t)-\tilde{x}$, then we could rewrite system (2) as

$$
D^{\alpha} y(t)=-C y(t)+B g(y(t))
$$

where $g(y(t))=f(y(t)+\tilde{x})-f(\tilde{x}), g(0)=0$, and for all $x, y \in \mathbb{H}, g(x)$ satisfies

$$
\left|g_{i}(y)-g_{i}(x)\right| \leq l_{i}|y-x|
$$

Construct a Lyapunov function as

$$
V(y(t))=y^{*} P y .
$$

Then the Lyapunov function satisfies

$$
\lambda_{\min }(P)\|y\|_{2}^{2} \leq V(y(t)) \leq \lambda_{\max }(P)\|y\|_{2}^{2} .
$$

Hence, the Lyapunov function satisfies the condition (6). By (22) and Lemma 8 we could obtain

$$
\begin{aligned}
& D^{\alpha} V(t, y(t)) \\
& \quad \leq y^{*}\left(-C^{*} P-P C+P B Q^{-1} B^{*} P+L Q L\right) y \\
& \quad \leq y^{*}\left(-\check{C}^{*} P-P \check{C}+P \tilde{B} Q^{-1} \tilde{B}^{*} P+L Q L\right) y \\
& \quad \leq \lambda_{\max }\left(-\check{C}^{*} P-P \check{C}+P \tilde{B} Q^{-1} \tilde{B}^{*} P+L Q L\right)\|y\|_{2}^{2} .
\end{aligned}
$$

From Lemma 4 and condition (23) we could see that $\lambda_{\max }\left(-\check{C}^{*} P-P \check{C}+P \tilde{B} Q^{-1} \tilde{B}^{*} P+L Q L\right)<$ 0 . It shows that the condition (7) in Lemma 6 is satisfied. Thus, the equilibrium point $\tilde{y}=0$ of (24) is globally Mittag-Leffler stable for all $C \in C_{I}, B \in B_{I}, I \in I_{I}$ by Lemma 6 and Assumption 2. Namely, the equilibrium point $\tilde{x}$ of system (2) is globally Mittag-Leffler stable for all $C \in C_{I}, B \in B_{I}, I \in I_{I}$. It follows from Definition 5 that system (2) is globally Mittag-Leffler robust stable.

Before presenting the result on the synchronization between the drive system (2) and the response system (4), we should design the form of the control law $u(t)$ in system (4). Let $e(t)=y(t)-\Upsilon x(t)$, where $\Upsilon \in \mathbb{H}^{n \times n}$ a projective coefficient matrix. Then from (2) and (4) we get the error system as follows:

$$
D^{\alpha} e(t)=-C y(t)+\Upsilon C x(t)+B f(y(t))-\Upsilon B f(x(t))+(E-\Upsilon) I+u(t) .
$$

Choose the control law $u(t)$ as

$$
\left\{\begin{array}{l}
u(t)=w(t)+v(t) \\
v(t)=(C \Upsilon-\Upsilon C) x(t)+\Upsilon B f(x(t))-B f(\Upsilon x(t))+(\Upsilon-E) I, \\
w(t)=-K(y(t)-\Upsilon x(t)),
\end{array}\right.
$$


where $K \in \mathbb{R}^{n \times n}$ is the coefficient matrix of the linear control $w(t)$. In fact, the control scheme (25) is a hybrid control, $v(t)$ is an open loop control, and $w(t)$ is a linear control.

Then we could get the following error system by using the control law (25):

$$
D^{\alpha} e(t)=(-K-C) e(t)+B h(e(t)),
$$

where $h(e(t))=f(y(t))-f(\Upsilon x(t))$.

Theorem 3 System (2) and system (4) realize global projective synchronization under the control law (25), if Assumption (1) holds and there exists a real diagonal matrix $Q$ and a Hermitian matrix $P>0$, such that control coefficient matrix $K$ satisfies

$$
\left(\begin{array}{cc}
(-K-C)^{*} P+P(-K-C)+L Q L & P B \\
B^{*} P & -Q
\end{array}\right)<0 .
$$

Proof It is clear that $\tilde{e}=0$ is an equilibrium point of system (26).

Construct a Lyapunov function as follows:

$$
V(e(t))=e^{*} P e .
$$

Then the following inequality holds:

$$
\lambda_{\min }(P)\|e\|_{2}^{2} \leq V(e(t)) \leq \lambda_{\max }(P)\|e\|_{2}^{2},
$$

which makes the condition (6) in Lemma 6 hold. From Assumption 1 and (26), we obtain

$$
h(e(t))=f(y(t))-f(\Upsilon x(t)) \leq L e(t) .
$$

Then, according to Lemmas 3, 7 and (29), we do the following calculations:

$$
\begin{aligned}
D^{\alpha} & V(e(t)) \\
& \leq\left(D^{\alpha} e(t)\right)^{*} P e(t)+[e(t)]^{*} P D^{\alpha} e(t) \\
& =[(-K-C) e(t)+B h(e(t))]^{*} P e(t)+[e(t)]^{*} P[(-K-C) e(t)+B h(e(t))] \\
& =e^{*}\left((-K-C)^{*} P+P(-K-C)\right) e+h^{*}(e) B^{*} P e+e^{*} P^{*} B h(e) \\
& \leq e^{*}\left((-K-C)^{*} P+P(-K-C)\right) e+e^{*} P B Q^{-1} B^{*} P e+h^{*}(e) Q h(e) \\
& \leq e^{*}\left((-K-C)^{*} P+P(-K-C)\right) e+e^{*} P B Q^{-1} B^{*} P e+e^{*} L Q L e \\
& =e^{*}\left((-K-C)^{*} P+P(-K-C)+P B Q^{-1} B^{*} P+L Q L\right) e \\
& \leq \lambda_{\max }\left((-K-C)^{*} P+P(-K-C)+P B Q^{-1} B^{*} P+L Q L\right)\|e\|_{2}^{2} .
\end{aligned}
$$

Based on Lemma 4 and condition (17), we get $\lambda_{\max }\left((-K-C)^{*} P+P(-K-C)+P B Q^{-1} B^{*} P+\right.$ $L Q L)<0$. It shows that the condition (7) in Lemma 6 is satisfied. Hence the equilibrium point $\tilde{e}=0$ of system (20) is globally Mittag-Leffler stable based on Lemma 6 . Therefore, system (2) and system (4) are globally projective synchronized under the control law (25) by Definitions 4 and 6 . 
Remark 4 In Theorems 1, 2 and 3, the LMI conditions for the global Mittag-Leffler stability, robust stability and projection synchronization of FQVNNs by Lyapunov stability theory were given, respectively. Unlike the methods in the existing literature [35, 41, 44], we considered FQVNNs directly instead of converting them into complex-valued or realvalued system.

\section{Some corollaries}

In this section, we will transform the quaternion-valued LMI conditions (17), (23) and (27) into complex-valued ones.

The quaternion-valued parameters $A, B$, and $P$ could be expressed by the following complex forms: $A=A_{1}+A_{2 J}, B=B_{1}+B_{2} J$ and $P=P_{1}+P_{2 J}$, where $A_{1}, A_{2}, B_{1}, B_{2}, P_{1}, P_{2} \in \mathbb{C}^{n \times n}$.

Corollary 1 Under Assumption 1, FQVNNs (2) have a unique equilibrium point $\tilde{x}$ which is globally Mittag-Leffler stable, if there exist a real positive diagonal matrix $Q$, a Hermitian matrix $P_{1} \in \mathbb{C}^{n \times n}$ and a skew-symmetric matrix $P_{2} \in \mathbb{C}^{n \times n}$ satisfying

$$
\left(\begin{array}{cc}
P_{1} & -P_{2} \\
\bar{P}_{2} & \bar{P}_{1}
\end{array}\right)>0, \quad\left(\begin{array}{cc}
M_{1} & -M_{2} \\
\bar{M}_{2} & \bar{M}_{1}
\end{array}\right)<0,
$$

where

$$
\begin{aligned}
& M_{1}=\left(\begin{array}{cc}
-C P_{1}-P_{1} C+L Q L & P_{1} B_{1}-P_{2} \bar{B}_{2} \\
B_{1}^{*} P_{1}+B_{2}^{T} \bar{P}_{2} & -Q
\end{array}\right), \\
& M_{2}=\left(\begin{array}{cc}
-C P_{2}-P_{2} \bar{C} & P_{1} B_{2}+P_{2} \bar{B}_{1} \\
B_{1}^{*} P_{2}-B_{2}^{T} \overline{P_{1}} & 0
\end{array}\right) .
\end{aligned}
$$

Proof By using Lemmas 1 and 2 as well as Theorem 1, the corollary can be proved straightforwardly.

Corollary 2 Under Assumptions 1 and 2, FQVNNs (2) have a unique equilibrium point and the equilibrium point is globally Mittag-Leffler robust stable, if there exist a real positive diagonal matrix $Q$, a Hermitian matrix $P_{1} \in C^{n \times n}$ and a skew-symmetric matrix $P_{2} \in C^{n \times n}$ such that the following LMI holds:

$$
\left(\begin{array}{cc}
P_{1} & -P_{2} \\
\bar{P}_{2} & \bar{P}_{1}
\end{array}\right)>0, \quad\left(\begin{array}{cc}
H_{1} & -H_{2} \\
\bar{H}_{2} & \bar{H}_{1}
\end{array}\right)<0,
$$

where

$$
\begin{aligned}
H_{1} & =\left(\begin{array}{cc}
-\check{C} P_{1}-P_{1} \check{C}+L Q L & P_{1} \tilde{B} \\
\tilde{B} * P_{1} & -Q
\end{array}\right), \\
H_{2} & =\left(\begin{array}{cc}
-\check{C} P_{2}-P_{2} \bar{C} & P_{2} \overline{\tilde{B}} \\
\tilde{B}^{*} P_{2} & 0
\end{array}\right) .
\end{aligned}
$$

Proof By using Lemmas 1 and 2 as well as Theorem 2, the corollary can be proved straightforwardly. 
Corollary 3 System (2) and system (4) realize global projective synchronization under the control law (25), if Assumption 1 holds and there exist a real positive diagonal matrix $Q$, a Hermitian matrix $P_{1} \in \mathbb{C}^{n \times n}$, and a skew-symmetric matrix $P_{2} \in \mathbb{C}^{n \times n}$, such that the control coefficient matrix $K$ satisfies

$$
\left(\begin{array}{cc}
P_{1} & -P_{2} \\
\bar{P}_{2} & \bar{P}_{1}
\end{array}\right)>0, \quad\left(\begin{array}{cc}
N_{1} & -N_{2} \\
\bar{N}_{2} & \bar{N}_{1}
\end{array}\right)<0,
$$

where

$$
\begin{aligned}
& N_{1}=\left(\begin{array}{cc}
N_{11} & P_{1} B_{1}-P_{2} \bar{B}_{2} \\
B_{1}^{*} P_{1}+B_{2}^{T} \bar{P}_{2} & -Q
\end{array}\right), \\
& N_{2}=\left(\begin{array}{cc}
(-K-C)^{*} P_{2}+P_{2}(-K-C) & P_{1} B_{2}+P_{2} \bar{B}_{1} \\
B_{1}^{*} P_{2}-B_{2}^{T} \bar{P}_{1} & 0
\end{array}\right),
\end{aligned}
$$

with $N_{11}=(-K-C)^{*} P_{1}+P_{1}(-K-C)+L Q L$.

Proof By using Lemmas 1 and 2 as well as Theorem 3, the corollary can be proved straightforwardly.

Remark 5 The LMIs (17), (23) and (27) are quaternion-valued in Theorems 1, 2 and 3, separately, which cannot be processed directly through the MATLAB LMI toolbox. By using Lemmas 1 and 2, these quaternion-valued LMIs are transformed into the complex-valued ones in the above three corollaries, which can be checked easily by the mathematical software MATLAB.

\section{Numerical examples}

In this section, there are three examples to demonstrate the effectiveness of our results.

Example 1 Consider the following 2-neuron FQVNNs:

$$
D^{\alpha} x(t)=-C x(t)+B f(x(t))+I
$$

where $\alpha=0.95, C=\operatorname{diag}\{2,2\}, f(x)=\sin \left(x_{0}\right)+\imath \sin \left(x_{1}\right)+\jmath \sin \left(x_{2}\right)+\kappa \sin \left(x_{3}\right), I=(0.2-0.2 \imath-$ $0.3 J+0.4 \kappa,-0.4+0.3 l+0.4 J-0.3 \kappa)$, and $B=\left(b_{i j}\right)_{2 \times 2}$, where

$$
\begin{aligned}
& b_{11}=-0.15-0.4 \imath-0.2 \jmath-0.5 \kappa, \\
& b_{12}=-0.5+0.2 \imath-0.4 \jmath+0.2 \kappa, \\
& b_{21}=-0.4+0.1 \imath-0.2 \jmath+0.25 \kappa, \\
& b_{22}=-0.6-0.1 \imath-0.3 \jmath-0.32 \kappa
\end{aligned}
$$

It is obvious that the parts of the parameter $B$ are

$$
B_{1}=\left(\begin{array}{cc}
-0.15-0.4 i & -0.5+0.2 i \\
-0.4+0.1 i & -0.6-0.1 i
\end{array}\right) \text {, }
$$



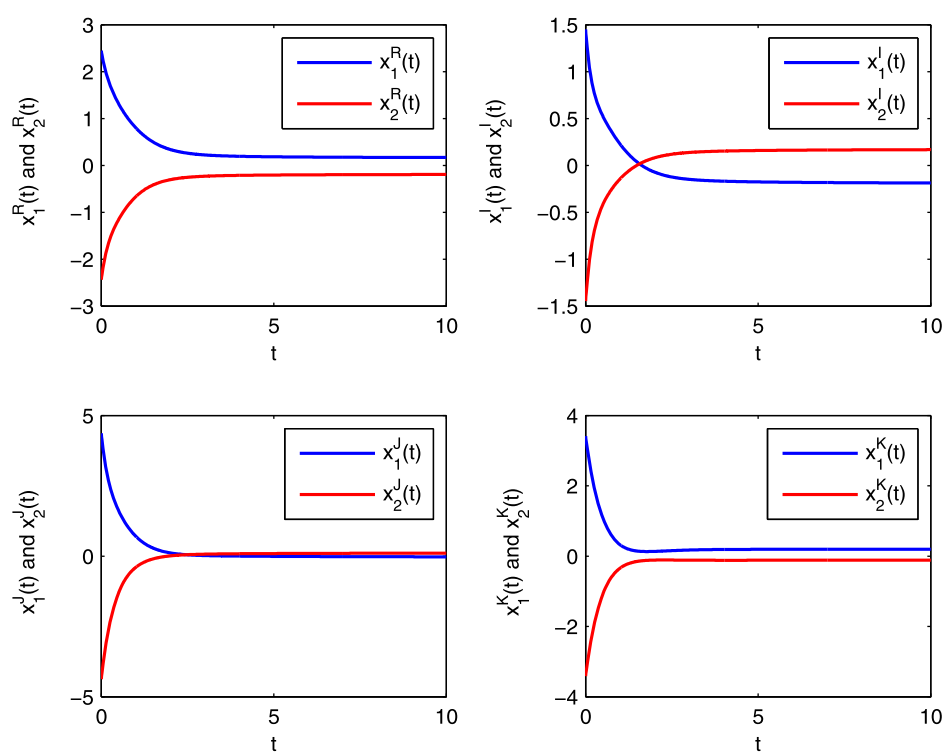

Figure 1 Transient states of four parts of FQVNNs (37)

$$
B_{2}=\left(\begin{array}{cc}
-0.2-0.5 i & -0.4+0.2 i \\
-0.2+0.25 i & -0.3-0.32 i
\end{array}\right) \text {. }
$$

By the YALMIP toolbox in MATLAB, we could find the following feasible solution to the LMIs (30):

$$
\begin{aligned}
& P_{1}=\left(\begin{array}{cc}
4.6860+0.0000 i & -0.1360+0.0166 i \\
-0.1360-0.0166 i & 4.7505+0.0000 i
\end{array}\right), \\
& P_{2}=\left(\begin{array}{cc}
0.0000+0.0000 i & -0.0231+0.0213 i \\
0.0231-0.0213 i & 0.0000+0.0000 i
\end{array}\right), \\
& Q=\left(\begin{array}{cc}
16.5914 & 0 \\
0 & 16.6145
\end{array}\right) .
\end{aligned}
$$

Therefore, system (37) has a unique equilibrium point and the unique equilibrium point is globally Mittag-Leffler stable by Corollary 1 . In the numerical simulation, the initial values are selected as $x_{10}=2.5+1.5 l+4.5 \jmath+3.5 \kappa$ and $x_{20}=-2.5-1.5 l-4.5 \jmath-3.5 \kappa$. Figure 1 depicts the time responses of four parts of the state variable of (37), which validates the effectiveness of Corollary 1.

Remark 6 In [44], the global Mittag-Leffler stability problem for FQVNNs is considered. If we use Theorem 3 in [44] to test the existence and Mittag-Leffler stability of the equilibrium point of FQVNNs (37) in Example 1 of our article. We need check $\hat{C}-|\hat{A}|$, defined in [44], is a non-singular M-matrix. By the parameters of FQVNNs (37), we could find that $\lambda(\hat{C}-|\hat{A}|)=-0.3368,2.6985,1.3715,1.4318,2.2030,1.9982,1.7668,1.8670$. Then $\hat{C}-|\hat{A}|$ is not a non-singular M-matrix. So we cannot check the existence and Mittag-Leffler stability of the equilibrium point of FQVNNs (37) in Example 1 by Theorem 3 in [44]. 
Example 2 Under Assumption 2, consider a 2-neuron FQVNN as follows:

$$
D^{\alpha} x(t)=-C x(t)+B f(x(t))+I,
$$

where

$$
\check{C}=\left(\begin{array}{ll}
1 & 0 \\
0 & 1
\end{array}\right), \quad \hat{C}=\left(\begin{array}{ll}
3 & 0 \\
0 & 3
\end{array}\right), \quad \check{B}=\left(\breve{b}_{i j}\right)_{2 \times 2}, \quad \hat{B}=\left(\hat{b}_{i j}\right)_{2 \times 2},
$$

where

$$
\begin{aligned}
& \check{b}_{11}=-0.32-0.24 \imath-0.24 J-0.18 \kappa, \\
& \check{b}_{12}=-0.256-0.144 \imath-0.192 \jmath-0.192 \kappa, \\
& \check{b}_{21}=-0.15-0.15 \imath-0.15 \jmath-0.15 \kappa, \\
& \check{b}_{22}=-0.384-0.288 \imath-0.288 \jmath-0.216 \kappa, \\
& \hat{b}_{11}=0.32+0.24 \imath+0.24 J+0.18 \kappa, \\
& \hat{b}_{12}=0.256+0.144 \imath+0.192 \jmath+0.192 \kappa, \\
& \hat{b}_{21}=0.15+0.15 \imath+0.15 J+0.15 \kappa, \\
& \hat{b}_{22}=0.384+0.288 \imath+0.288 \jmath+0.216 \kappa .
\end{aligned}
$$

We could get the following matrix by matrices $\breve{B}$ and $\hat{B}$ :

$$
\tilde{B}=\left(\begin{array}{ll}
0.5 & 0.4 \\
0.3 & 0.6
\end{array}\right)
$$

Letting $L=\operatorname{diag}\{0.3,0.3\}$, we could get the following feasible solutions of LMI (33) in Corollary 2 by suing the YALMIP toolbox in MATLAB:

$$
P_{1}=\left(\begin{array}{cc}
11.9850 & -1.5844 \\
-1.5844 & 11.8216
\end{array}\right), \quad P_{2}=\left(\begin{array}{ll}
0 & 0 \\
0 & 0
\end{array}\right), \quad Q=\left(\begin{array}{cc}
21.5872 & 0 \\
0 & 21.5580
\end{array}\right)
$$

Thus the conditions in Corollary 2 are satisfied. Then system (38) has a unique equilibrium point and the equilibrium point is globally robust stable.

Now we consider a special model of this example. We choose the following fixed network parameters: $C=\operatorname{diag}\{1,2\}, B=\left(b_{i j}\right)_{2 \times 2}, f(x)=\sin \left(x_{0}\right)+\imath \sin \left(x_{1}\right)+j \sin \left(x_{2}\right)+\kappa \sin \left(x_{3}\right)$, and $I=(0.2-0.2 \imath-0.3 \jmath+0.4 \kappa,-0.4+0.3 \imath+0.4 \jmath-0.3 \kappa)$, where

$$
\begin{aligned}
& b_{11}=0.3-0.2 \imath+0.15 \jmath-0.1 \kappa, \\
& b_{12}=-0.25+0.14 \imath-0.15 \jmath+0.15 \kappa, \\
& b_{21}=0.1-0.1 \imath-0.12 \jmath+0.13 \kappa, \\
& b_{22}=-0.38-0.25 \imath+0.25 \jmath-0.2 \kappa .
\end{aligned}
$$

By numerical simulation in MATLAB, we get the four parts of the states decided by the considered system, which initial conditions are chosen by 10 random constant quaternion- 

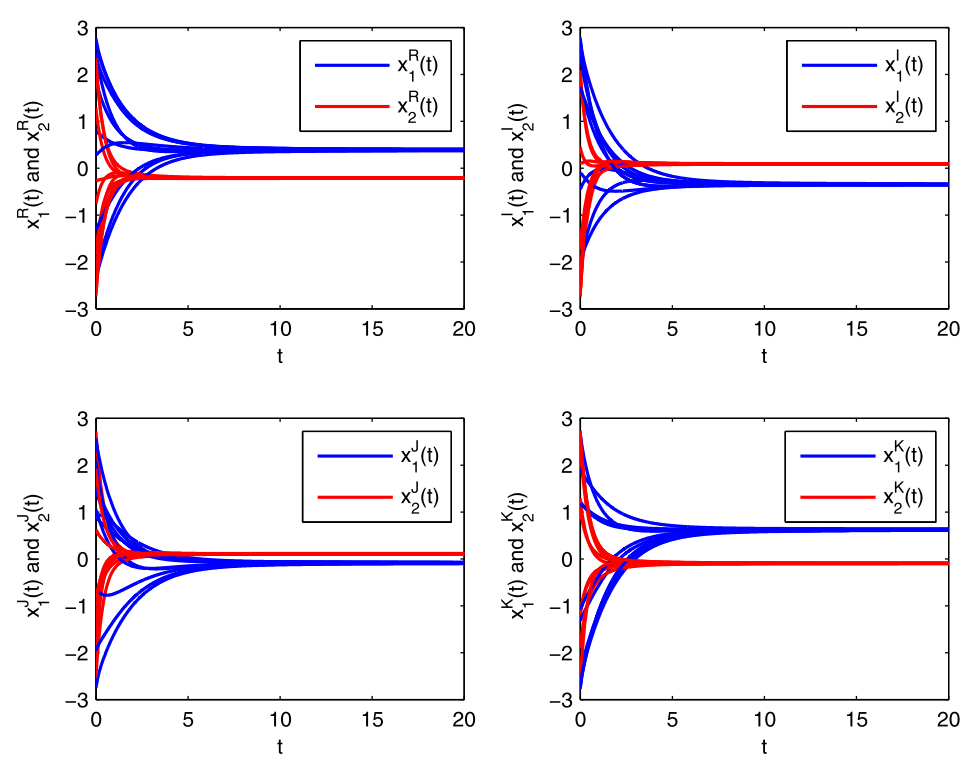

Figure 2 The four parts of the state trajectories for system (38)

valued vectors. Moreover, it is showed in Fig. 2, which depicts that each neuron state converges to the stable state.

Example 3 Consider the following 2-neuron FQVNNs as the drive system:

$$
D^{\alpha} x(t)=-C x(t)+B f(x(t))+I,
$$

where $\alpha=0.95, C=\operatorname{diag}\{1,1\}, f(x)=\tanh \left(x_{0}\right)+\imath \tanh \left(x_{1}\right)+J \tanh \left(x_{2}\right)+\kappa \tanh \left(x_{3}\right), I=(0.2-$ $0.2 \imath-0.3 \jmath+0.4 \kappa,-0.4+0.3 \imath+0.4 J-0.3 \kappa)$ and $B=\left(b_{i j}\right)_{2 \times 2}$

$$
\begin{aligned}
& b_{11}=1.2-1.2 \imath+1.2 \jmath-0.9 \kappa, \\
& b_{12}=1.5+0.68 \imath-1.4 \jmath+1.2 \kappa, \\
& b_{21}=1.1+0.79 \imath+0.9 \jmath+1.5 \kappa, \\
& b_{22}=-0.8-0.83 \imath+1.4 \jmath+1.4 \kappa .
\end{aligned}
$$

Then the corresponding response system is showed as follows:

$$
D^{\alpha} y(t)=-C y(t)+B f(y(t))+I+u(t)
$$

where $\alpha=0.95, y(t) \in \mathbb{H}^{n}, f(y)=\tanh \left(y_{0}\right)+\imath \tanh \left(y_{1}\right)+j \tanh \left(y_{2}\right)+\kappa \tanh \left(y_{3}\right), C, B$ and $I$ are the same as that in (39), the control law $u(t)$ in (40) is designed as (25). Therefore, the error system between drive system (39) and response system (40) is described by

$$
D^{\alpha} e(t)=(-K-C) e(t)+B g(e(t)) \text {, }
$$

where $g(e(t))=f(y(t))-\Upsilon f(x(t))$. 
We choose $L=\operatorname{diag}\{0.3,0.3\}$, and

$$
K=\left(\begin{array}{ll}
1 & 1 \\
2 & 1
\end{array}\right) .
$$

It is easy to get the parts of the parameter $B$ :

$$
\begin{aligned}
& B_{1}=\left(\begin{array}{cc}
1.2-1.2 i & 1.5+0.68 i \\
1.1+0.79 i & -0.8-0.83 i
\end{array}\right), \\
& B_{2}=\left(\begin{array}{cc}
1.2-0.9 i & -1.4+1.2 i \\
0.9+1.5 i & 1.4+1.4 i
\end{array}\right) .
\end{aligned}
$$

By the YALMIP toolbox in MATLAB, we could find the following feasible solution to the LMIs (36):

$$
\begin{aligned}
& P_{1}=\left(\begin{array}{cc}
1.2985+0.0000 i & -0.0768+0.0231 i \\
-0.0768-0.0231 i & 1.2177+0.0000 i
\end{array}\right), \\
& P_{2}=\left(\begin{array}{cc}
0.0000+0.0000 i & -0.0051+0.1557 i \\
0.0051-0.1557 i & 0.0000+0.0000 i
\end{array}\right), \\
& Q=\left(\begin{array}{cc}
10.4014 & 0 \\
0 & 10.3719
\end{array}\right) .
\end{aligned}
$$

Hence, system (41) has a unique equilibrium point and the unique equilibrium point is globally Mittag-Leffler stable by Corollary 3. In other words, system (39) and system (40) are globally asymptotically projective synchronized. In numerical simulation, the initial values for the drive system (39) are selected as $x_{10}=1.5+0.5 \imath+2.5 J+3 \kappa$ and $x_{20}=-1.5-$ $0.5 \imath-2.5 \jmath-3 \kappa$, and the initial values for the drive system (40) are selected as $y_{10}=-2.5-$ $3 \imath-3 \jmath-2 \kappa$ and $y_{20}=2.5+3 \imath+3 \jmath+2 \kappa$.

At first, we consider the global Mittag-Leffler complete projective synchronization between system (39) and (40). Namely we choose the projective coefficient matrix

$$
\Upsilon=\left(\begin{array}{ll}
1 & 0 \\
0 & 1
\end{array}\right)
$$

Figures 3 and 4 show the time response of four parts of state of drive system (39) and response system (40) with control. The phase plot of drive-response system (39) and (40) with control is shown in Figs. 5. Moreover, Fig. 6 shows the time responses of four parts of state variable of the error system (41). In addition, Figs. 7 and 8 show the time response of four parts of state of drive system (39) and response system (40) without control. Figure 9 shows the phase plot of drive-response system (39) and (40) without control.

In the same way, we choose the projective coefficient matrix

$$
\Upsilon=\left(\begin{array}{cc}
-1 & 0 \\
0 & -1
\end{array}\right) \text {. }
$$



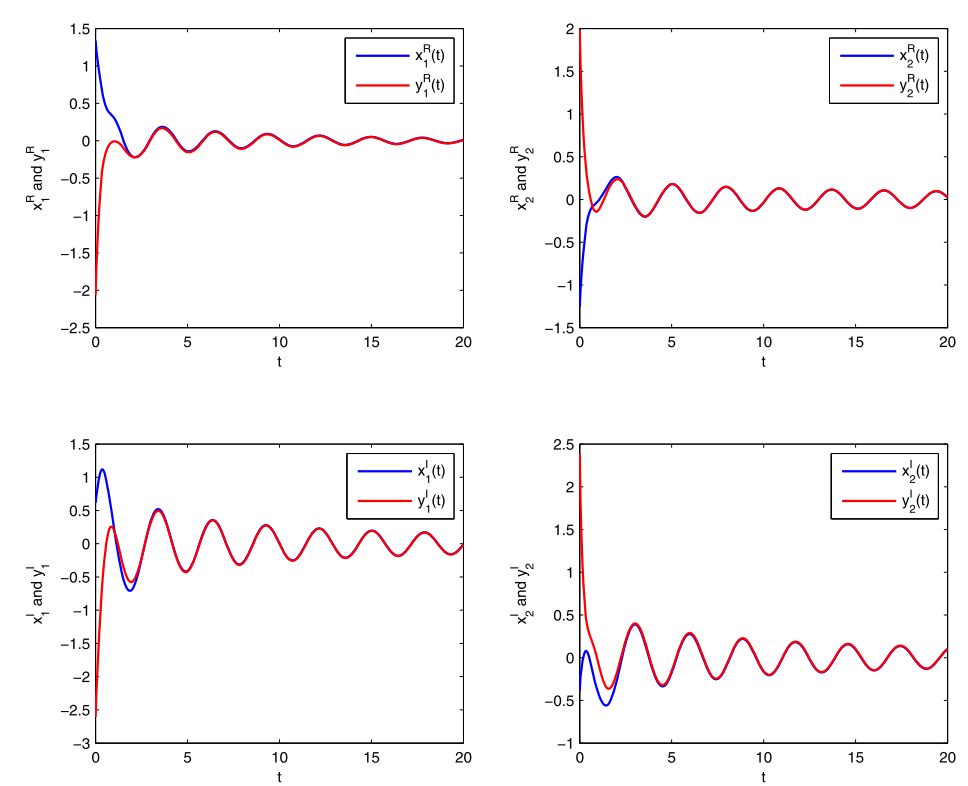

Figure 3 Transient states of the first and second parts drive-response systems (39) and (40) with the projective matrix (42) under control
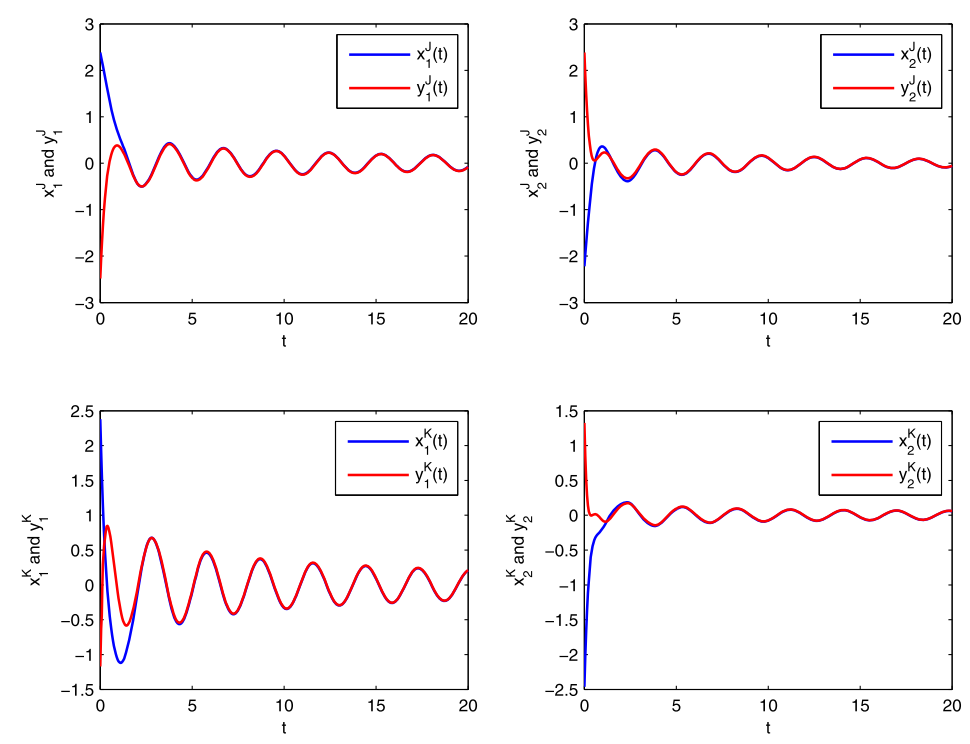

Figure 4 Transient states of the third and fourth parts drive-response systems (39) and (40) with the projective matrix (42) under control

Then system (39) and system (40) realize global Mittag-Leffler projective anti-synchronization. Figures 10 and 11 show the time response of four parts of states of drive system (39) and response system (40) with the projective coefficient matrix (43) under control. Figure 12 shows the phase plot of drive-response system (39) and (40) with the projective coefficient matrix (43) under control. Moreover, Fig. 13 shows the time response of four parts of states variable of error system (41). 

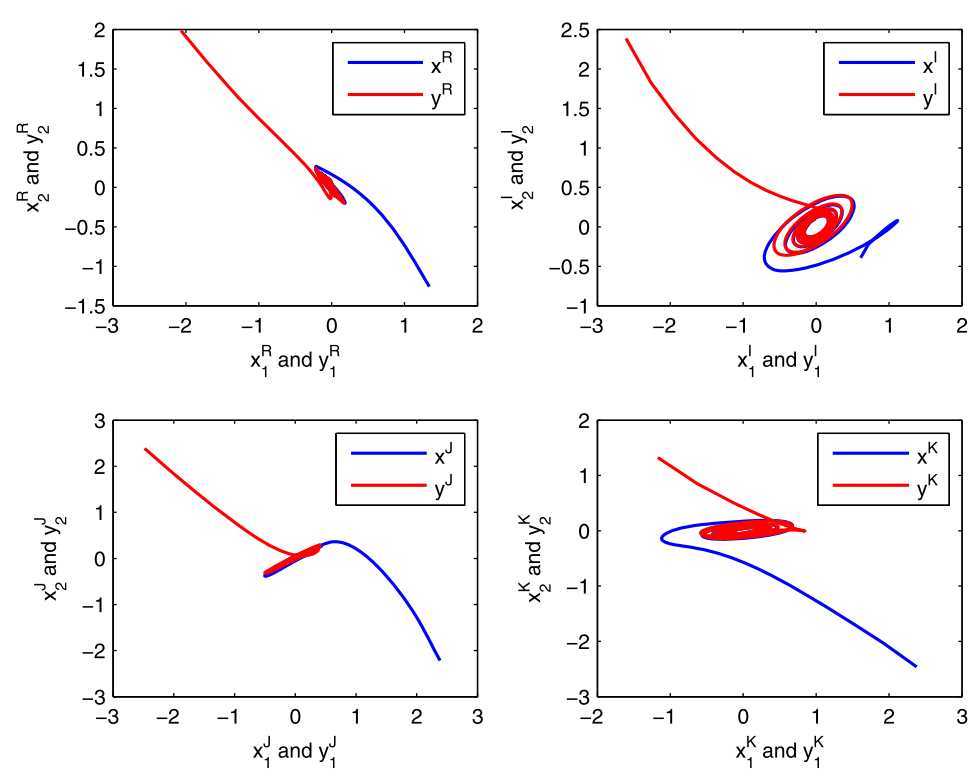

Figure 5 Phase plot of drive-response systems (39) and (40) with the projective matrix (42) under control
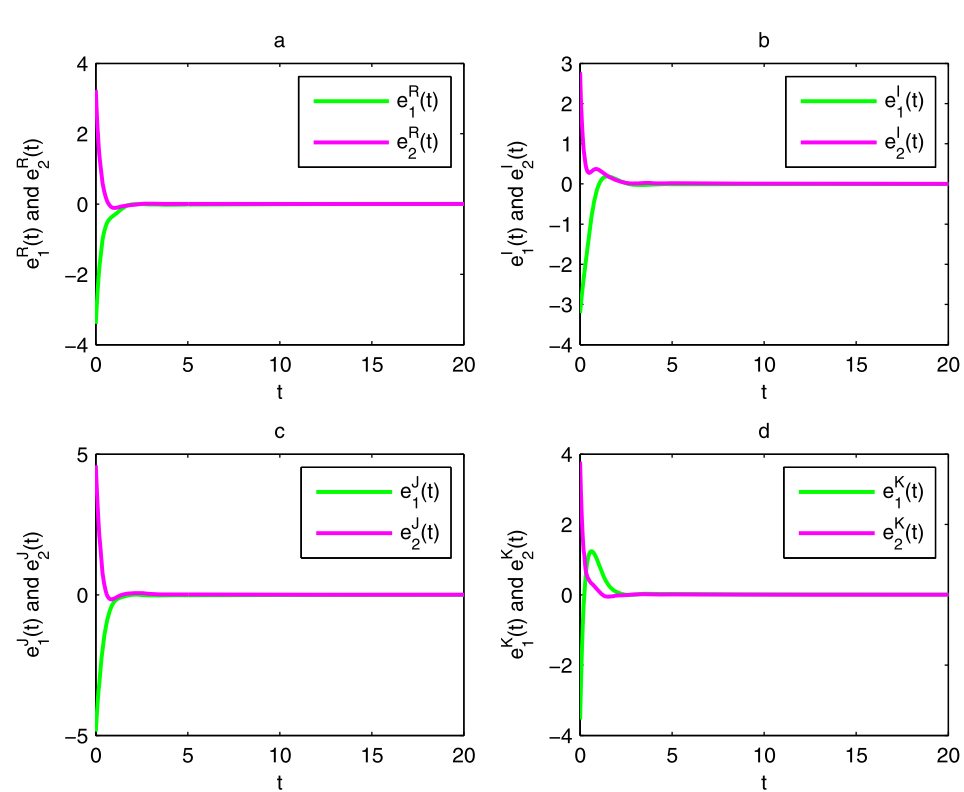

Figure 6 Transient states of four parts of error system (41) between (39) and (40) with the projective matrix (42) under control

Lastly, we consider the global projection synchronization. Then we choose the projective coefficient matrix

$$
\Upsilon=\left(\begin{array}{cc}
-1 & 0.7 \\
0.5 & 2
\end{array}\right)
$$



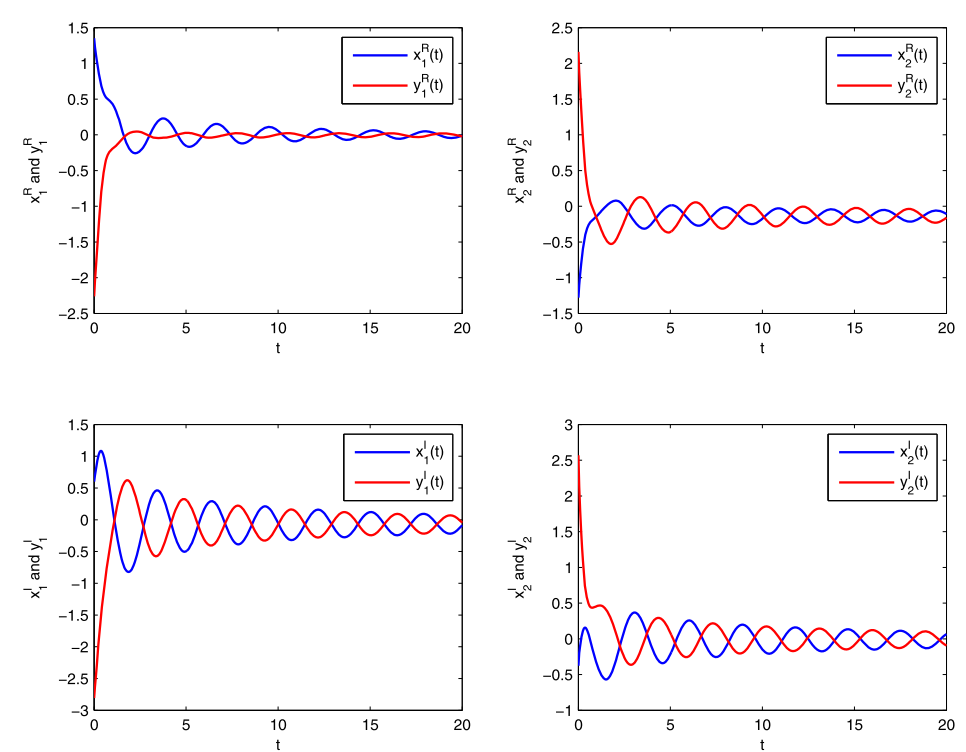

Figure 7 Transient states of the first and second parts of drive-response systems (39) and (40) without control
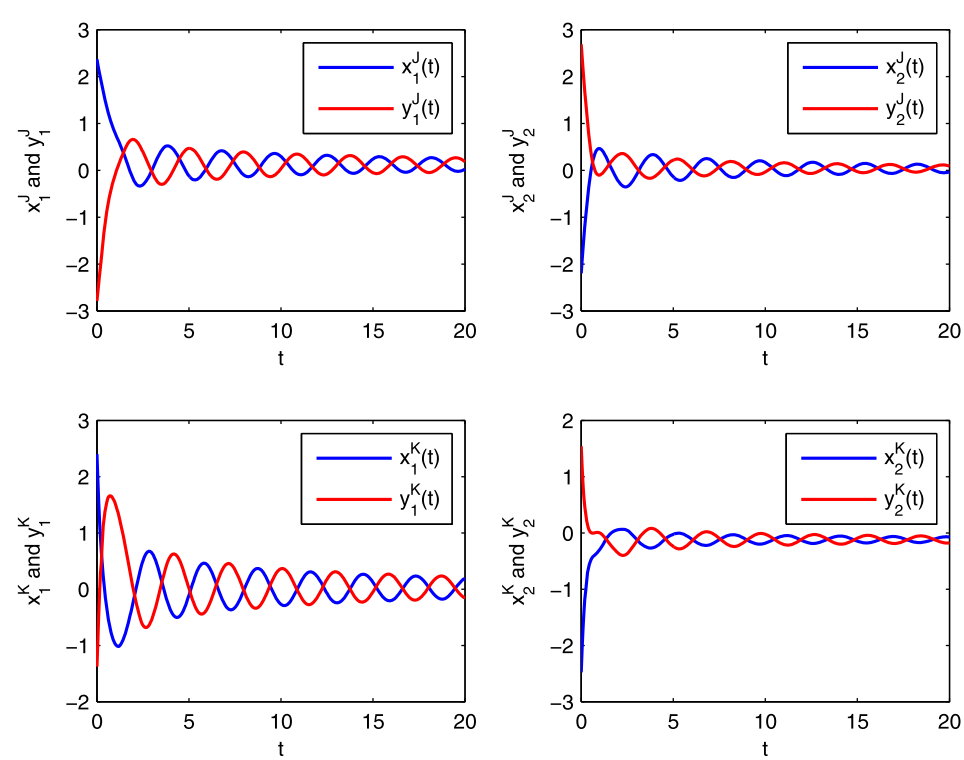

Figure 8 Transient states of the third and fourth parts of drive-response systems (39) and (40) without control

Figures 14 and 15 show the time response of four parts of states of drive system (39) and response system (40) with the projective coefficient matrix (44) under control. Figure 16 shows the phase plot of drive-response system (39) and (40) with the projective coefficient matrix (44) under control. Figure 17 shows the time response of four parts of states variable of error system (41).

Remark 7 When we choose $\Upsilon=\left(\begin{array}{ll}1 & 0 \\ 0 & 1\end{array}\right)$ and $K=\left(\begin{array}{ll}4 & 0 \\ 0 & 4\end{array}\right)$, the controller is the same as the one in the literature [44]. By using the LMI condition (3) in this paper we could calculate that 

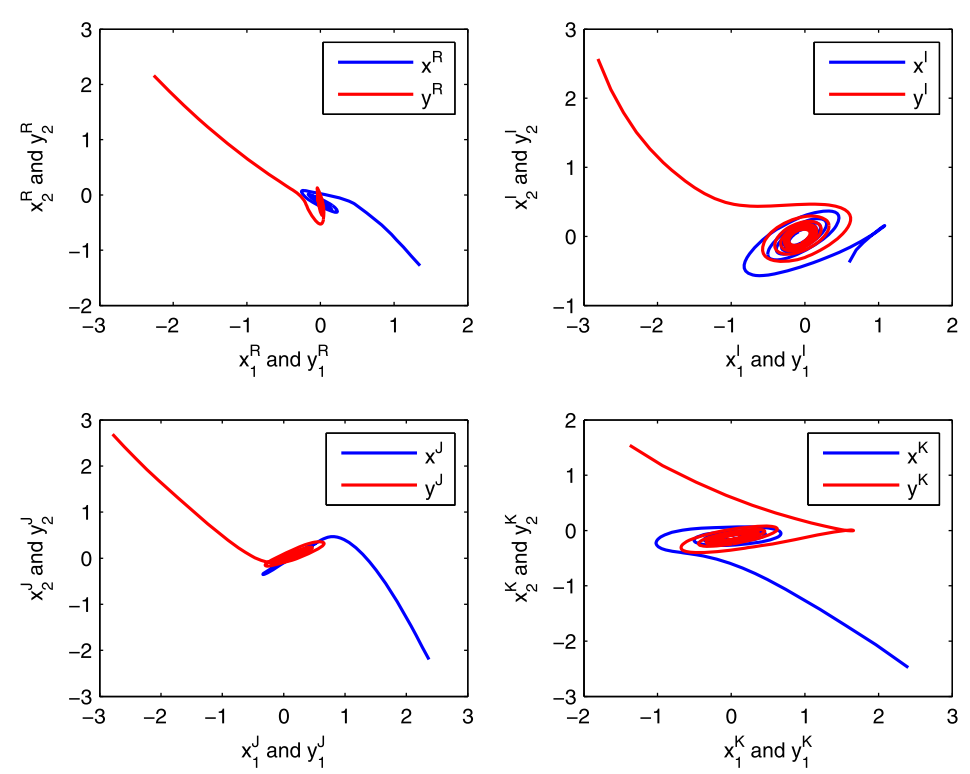

Figure 9 Phase plot of drive-response systems (39) and (40) without control
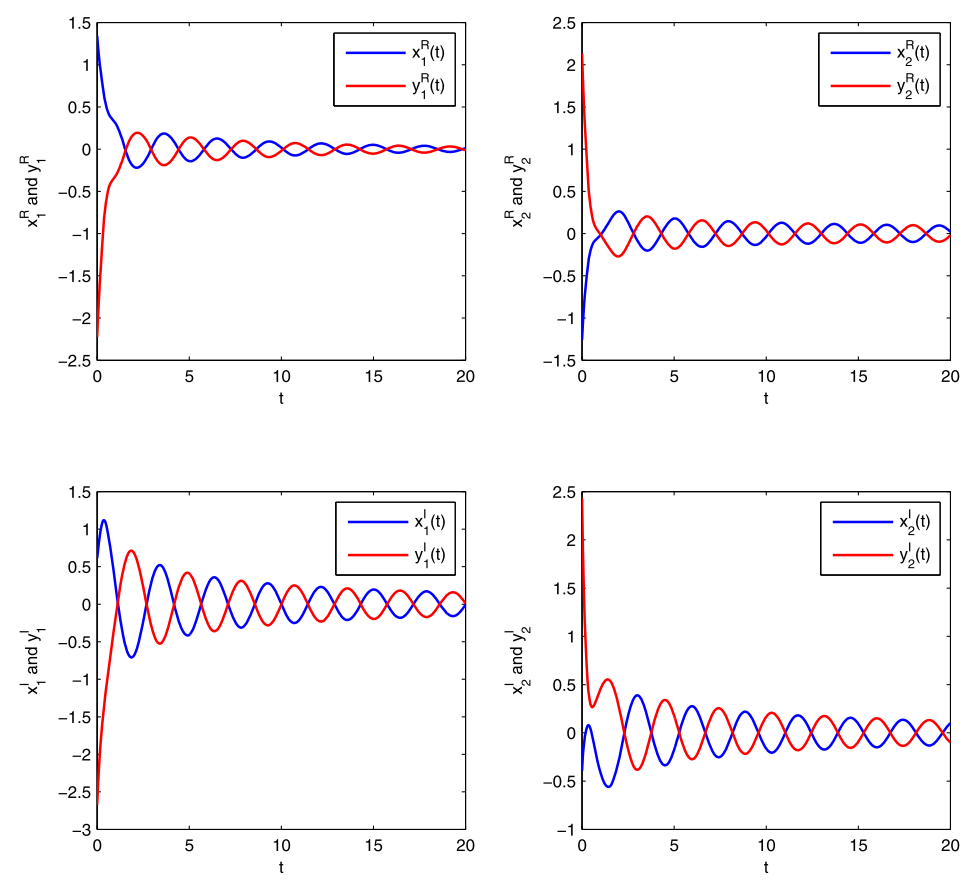

Figure 10 Transient states of the first and second parts drive-response systems (39) and (40) with the projective matrix (43) under control

$\lambda(N)=-16.0622,-16.0622,-15.5803,-15.5803,-9.7406,-9.7406,-7.9506,-7.9506$. We find system (39) and (40) will be complete synchronization. But when we use Theorem 6 

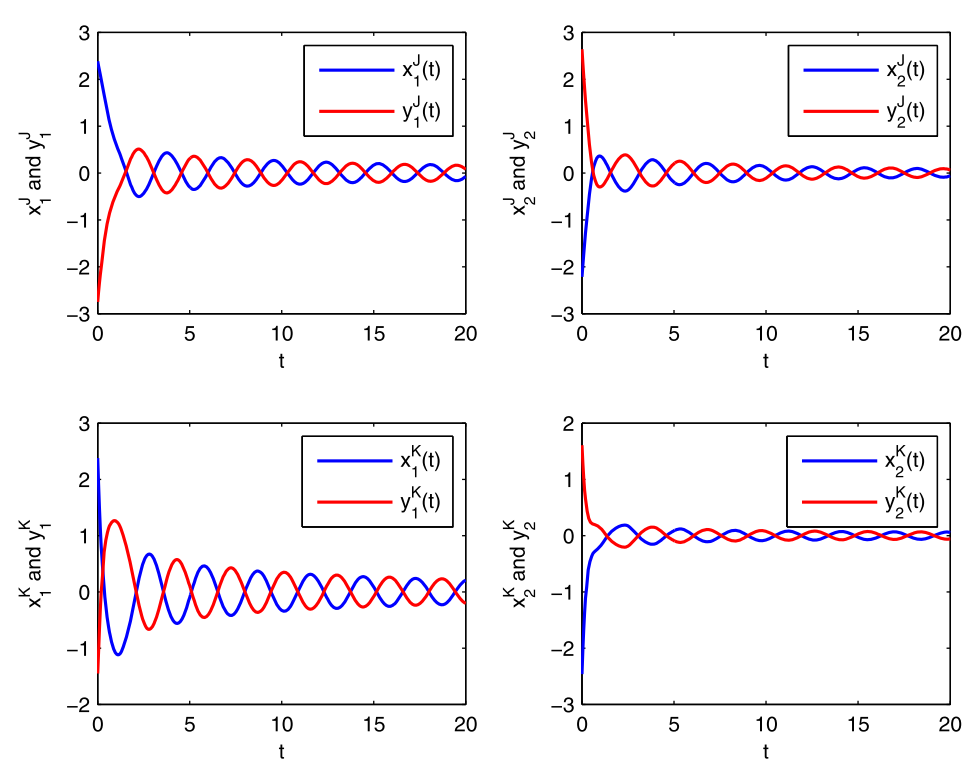

Figure 11 Transient states of the third and fourth parts drive-response systems (39) and (40) with the projective matrix (43) under control
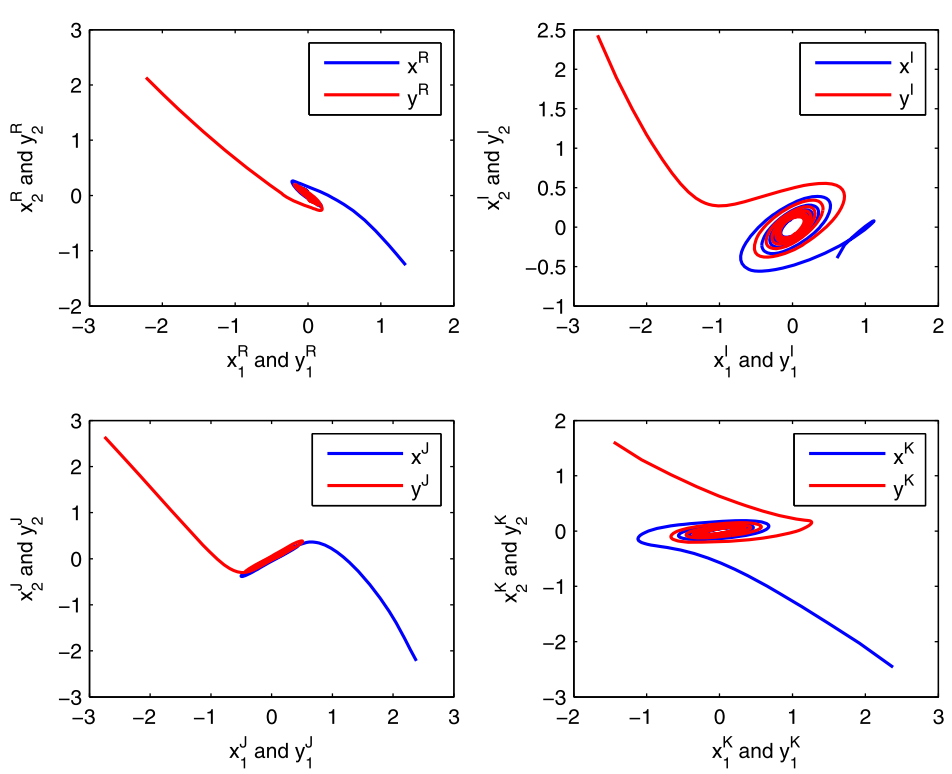

Figure 12 Phase plot of drive-response systems (39) and (40) with the projective matrix (43) under control

in [44] to test the synchronization, we cannot find any positive constant $\rho$ such that

$$
\begin{aligned}
\lambda_{1}= & \min _{1 \leq p \leq n}\left\{2\left(c_{p}+\sigma_{p}\right)-\sum_{q=1}^{n} \rho^{-1}\left(\left|a_{p q}^{R}\right|+\left|a_{p q}^{I}\right|+\left|a_{p q}^{I}\right|+\left|a_{p q}^{K}\right|\right)\right. \\
& \left.-\sum_{q=1}^{n} \rho\left(\left|a_{q p}^{R}\right|+\left|a_{q p}^{I}\right|+\left|a_{q p}^{J}\right|+\left|a_{q p}^{K}\right|\right)\right\}>0 .
\end{aligned}
$$



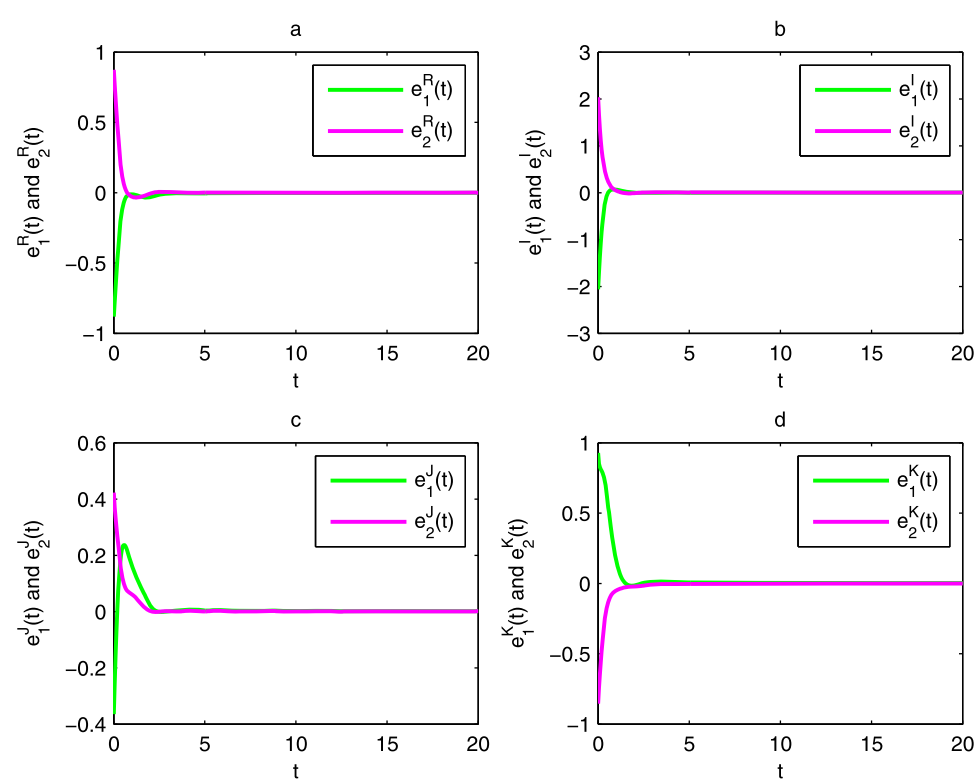

Figure 13 Transient states of four parts of error system (41) between (39) and (40) with the projective matrix (43) under control
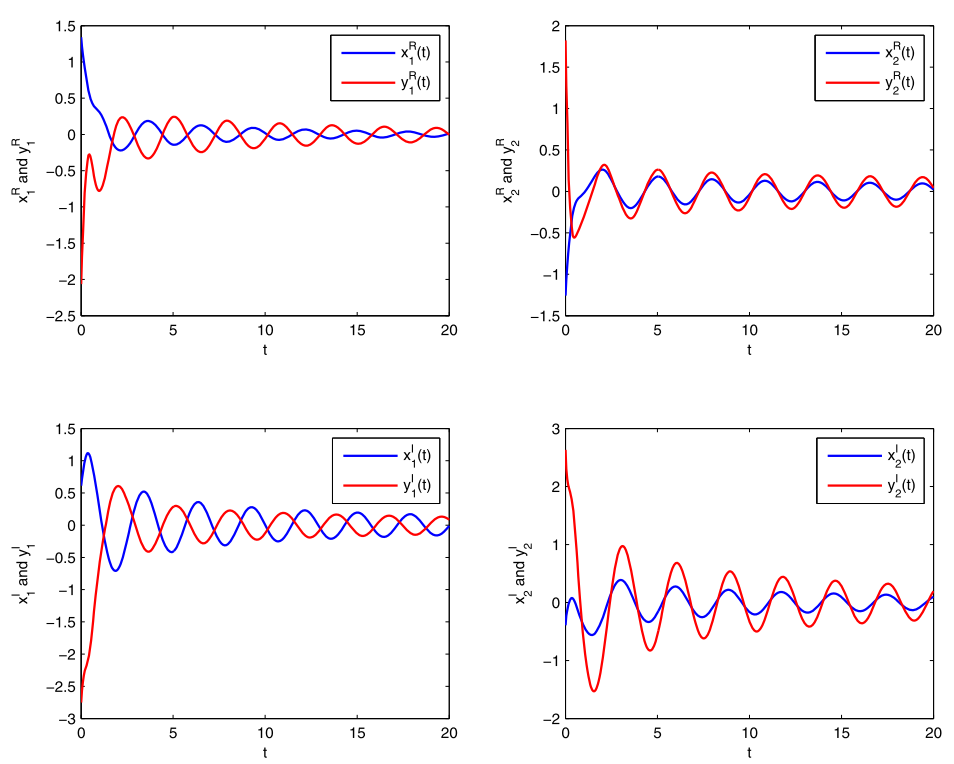

Figure 14 Transient states of the first and second parts drive-response systems (39) and (40) with the projective matrix (44) under control

In fact, for all $\rho>0$, we have $2\left(c_{1}+\sigma_{1}\right)-\sum_{q=1}^{n} \rho^{-1}\left(\left|a_{1 q}^{R}\right|+\left|a_{1 q}^{I}\right|+\left|a_{1 q}^{I}\right|+\left|a_{1 q}^{K}\right|\right)-\sum_{q=1}^{n} \rho\left(\left|a_{q 1}^{R}\right|+\right.$ $\left.\left|a_{q 1}^{I}\right|+\left|a_{q 1}^{J}\right|+\left|a_{q 1}^{K}\right|\right)=10-9.28 \rho^{-1}-8.79 \rho \leq-7.58$ and $2\left(c_{2}+\sigma_{2}\right)-\sum_{q=2}^{n} \rho^{-1}\left(\left|a_{2 q}^{R}\right|+\left|a_{2 q}^{I}\right|+\right.$ $\left.\left|a_{2 q}^{J}\right|+\left|a_{2 q}^{K}\right|\right)-\sum_{q=1}^{n} \rho\left(\left|a_{q 2}^{R}\right|+\left|a_{q 2}^{I}\right|+\left|a_{q 2}^{J}\right|+\left|a_{q 2}^{K}\right|\right)=10-8.72 \rho^{-1}-9.21 \rho \leq-7$, 44. So Theorem 6 in [44] cannot check system (39) and (40) realizes complete synchronization. 

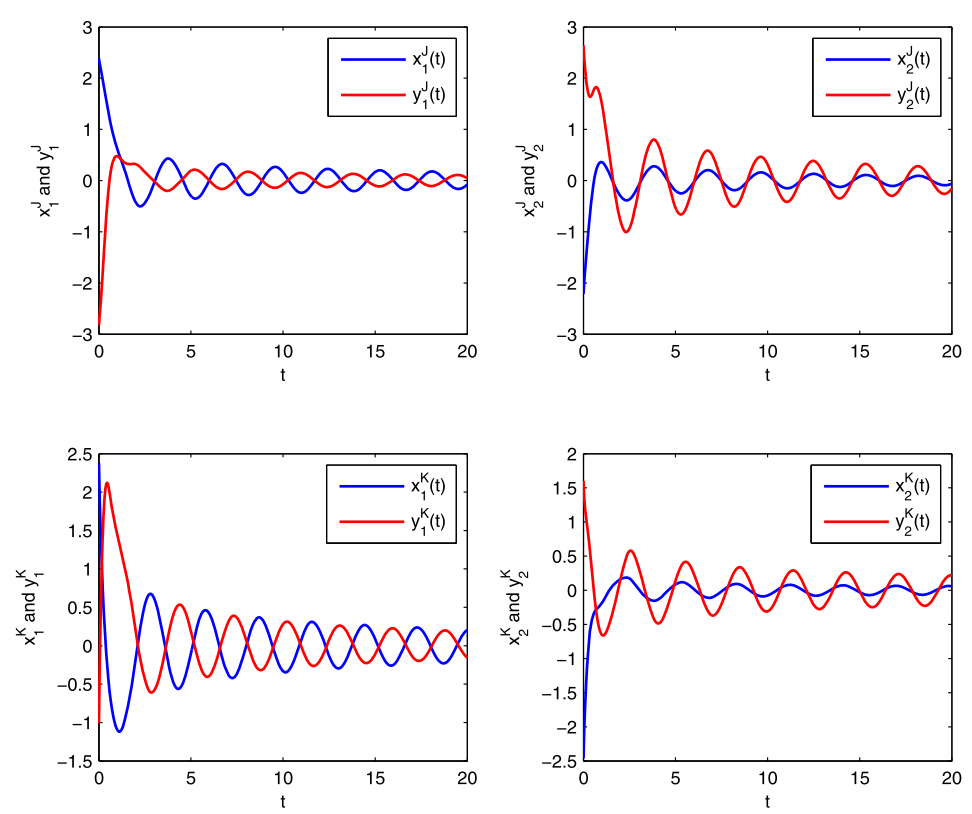

Figure 15 Transient states of the third and fourth parts drive-response systems (39) and (40) with the projective matrix (44) under control
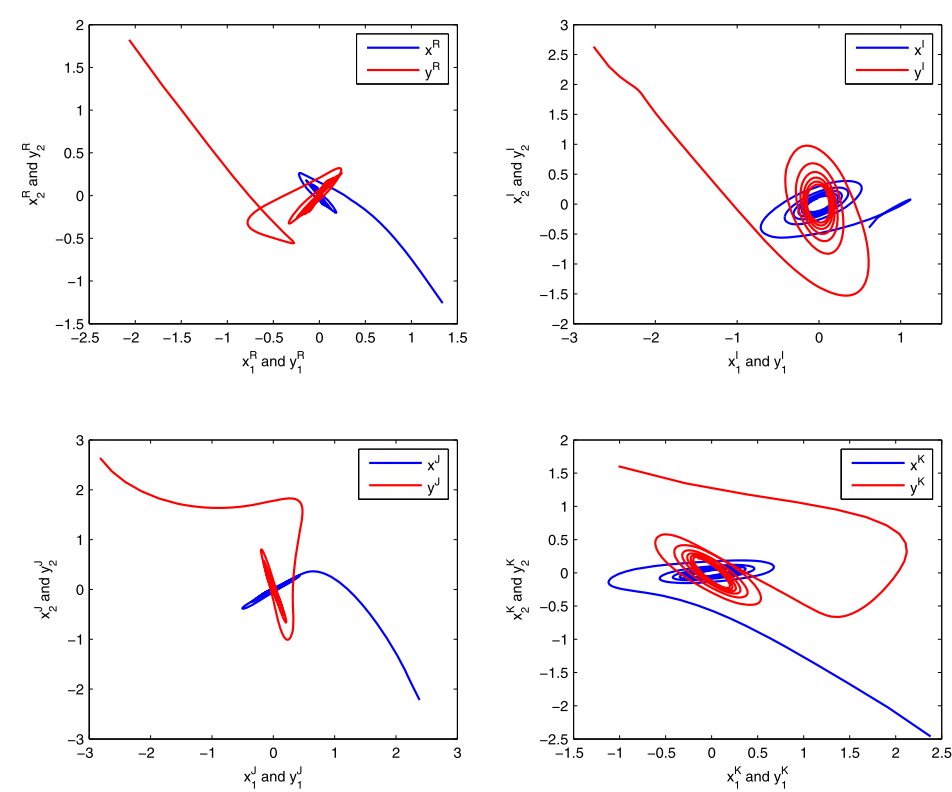

Figure 16 Phase plot of drive-response systems (39) and (40) with the projective matrix (44) under control

\section{Conclusions}

In this paper, some dynamical behaviors, including global Mittag-Leffler stability, robust stability and projection synchronization, for FQVNNs are studied. A LMI condition is given for Mittag-Leffler stability of FQVNNs by Lyapunov stability theory and homeomorphic mapping theory. Based on this, a modulus inequality technique of quaternions is used to study the robust stability of FQVNNs, and obtain a sufficient LMI condition 

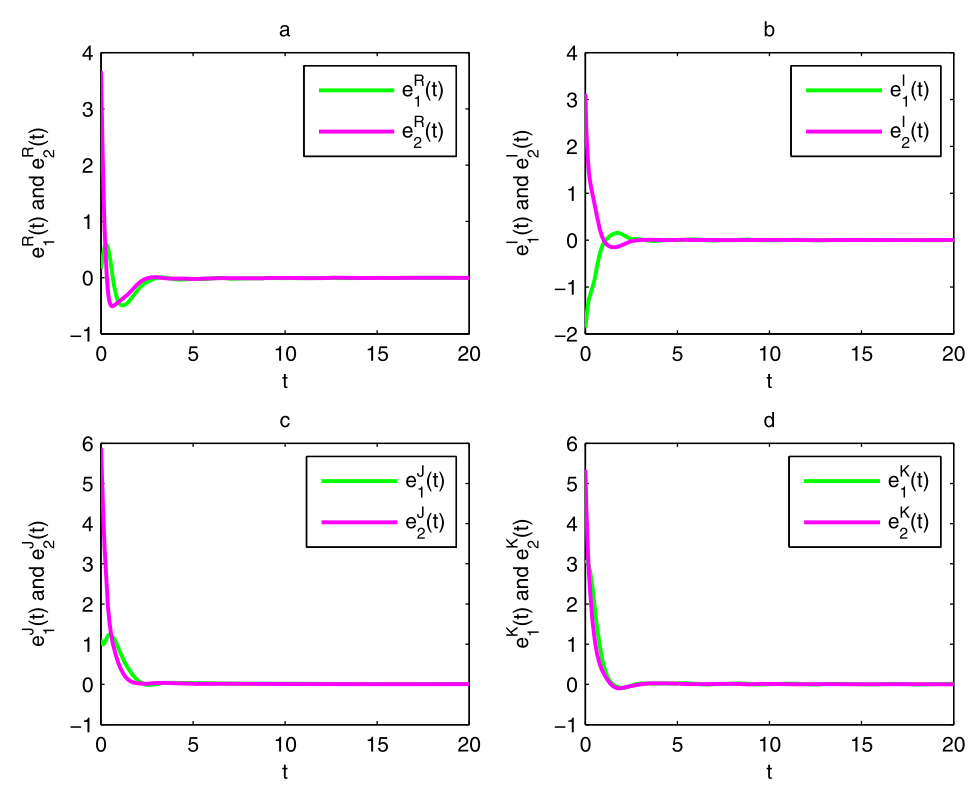

Figure 17 Transient states of four parts of error system (41) between (39) and (40) with the projective matrix (44) under control

for robust stability of FQVNNs. Moreover, the LMI condition for global Mittag-Leffler projection synchronization between FQVNNs is also given by the application of the projective synchronization theory. In addition, two corollaries for Mittag-Leffler stability and projective synchronization are given to make the validity of the conditions can be tested by the mathematical software MATLAB. Finally, three examples are given to substantiate the effectiveness of the theoretical results.

\section{Funding}

This work was supported in part by the National Natural Science Foundation of China under Grant 61773004, the Natural Science Foundation of Chongqing under Grants cstc2017jcyjAX0082 and cstc2018jcyjAX0606, the Science and Technology Research Program of Chongqing Municipal Education Commission under Grant KJQN201800733, the Innovation Project for Returned Overseas Scholars of Chongqing under Grant CX2018115, and the Promotion Project for Young and Middle-aged Teachers' Basic Scientific Research Ability in Colleges and Universities of GuangXi under Grant 2019KY0382.

\section{Competing interests}

The authors declare that they have no competing interests.

\section{Authors' contributions}

All authors conceived of the study, participated in its design and coordination, read and approved the final manuscript.

\section{Publisher's Note}

Springer Nature remains neutral with regard to jurisdictional claims in published maps and institutional affiliations.

Received: 20 March 2019 Accepted: 19 June 2019 Published online: 04 July 2019

\section{References}

1. Podlubny, I.: Fractional Differential Equations. Academic Press, San Diego (1999)

2. Kilbas, A.A., Srivastava, H.M., Trujillo, J.J.: Theory and Applications of Fractional Differential Equations. Elsevier, Amsterdam (2006)

3. Soczkiewicz, E.: Application of fractional calculus in the theory of viscoelasticity. Mol. Quantum Acoust. 23, 397-404 (2002)

4. Tripathi, D., Pandey, S.K., Das, S.: Peristaltic flow of viscoelastic fluid with fractional Maxwell model through a channel. Appl. Math. Comput. 215(10), 3645-3654 (2010) 
5. Szabo, T.L., Wu, J.: A model for longitudinal and shear wave propagation in viscoelastic media. J. Acoust. Soc. Am. 107(5), 2437-2446 (2000)

6. Kilbas, A.A., Marzan, S.A.: Nonlinear differential equations with the Caputo fractional derivative in the space of continuously differentiable functions. Differ. Equ. 41(1), 84-89 (2005)

7. Duarte Isfer, L.A., Kaminski Lenzi, E., Marcelo Teixeira, G., Kaminski Lenzi, M.: Fractional control of an industrial furnace. Acta Scientiarum. Technology 32(3) (2010)

8. Metzler, R., Klafter, J.: The random walk's guide to anomalous diffusion: a fractional dynamics approach. Phys. Rep. $339(1), 1-77(2000)$

9. Henry, B.I., Wearne, S.L.: Existence of Turing instabilities in a two-species fractional reaction-diffusion system. SIAM J. Appl. Math. 62(3), 870-887 (2006)

10. Sugimoto, N.: Burgers equation with a fractional derivative; hereditary effects on nonlinear acoustic waves. J. Fluid Mech. 225, 631-653 (1991)

11. Raja, M.A.Z., Khan, J.A., Qureshi, I.M.: A new stochastic approach for solution of Riccati differential equation of fractional order. Ann. Math. Artif. Intell. 60(3-4), 229-250 (2010)

12. Pan, J., Liu, X., Xie, W.: Exponential stability of a class of complex-valued neural networks with time-varying delays. Neurocomputing 164, 293-299 (2015)

13. Kaslik, E., Sivasundaram, S.: Nonlinear dynamics and chaos in fractional-order neural networks. Neural Netw. 32, 245-256 (2012)

14. Rakkiyappan, R., Udhayakumar, K., Velmurugan, G., Cao, J., Alsaedi, A.: Stability and Hopf bifurcation analysis of fractional-order complex-valued neural networks with time delays. Adv. Differ. Equ. 2017, 225 (2017)

15. Wan, L., Wu, A.: Mittag-Leffler stability analysis of fractional-order fuzzy Cohen-Grossberg neural networks with deviating argument. Adv. Differ. Equ. 2017(1), 308 (2017)

16. $\mathrm{Yu}, \mathrm{J}$., $\mathrm{Hu}, \mathrm{C} .$, Jiang, $\mathrm{H} .: \alpha$-stability and $\alpha$-synchronization for fractional-order neural networks. Neural Netw. 35, $82-87$ (2012)

17. Yang, X., Song, Q., Liu, Y., Zhao, Z.: Finite-time stability analysis of fractional-order neural networks with delay. Neurocomputing 152, 19-26 (2015)

18. Liu, Y., Xu, P., Lu, J., Liang, J.: Global stability of Clifford-valued recurrent neural networks with time delays. Nonlinear Dyn. 84(2), 767-777 (2016)

19. Banu, L.J., Balasubramaniam, P., Ratnavelu, K.: Robust stability analysis for discrete-time uncertain neural networks with leakage time-varying delay. Neurocomputing 151, 808-816 (2015)

20. Wu, A., Liu, L., Huang, T., Zeng, Z.: Mittag-Leffler stability of fractional-order neural networks in the presence of generalized piecewise constant arguments. Neural Netw. 85, 118-127 (2017)

21. Ding, Z., Shen, Y., Wang, L.: Global Mittag-Leffler synchronization of fractional-order neural networks with discontinuous activations. Neural Netw. 73, 77-85 (2016)

22. Zhang, S., Yu, Y., Yu, J.: LMI conditions for global stability of fractional-order neural networks. IEEE Trans. Neural Netw. Learn. Syst. 28(10), 2423-2433 (2017)

23. Bao, H.-B., Cao, J.-D.: Projective synchronization of fractional-order memristor-based neural networks. Neural Netw. $63,1-9(2015)$

24. Rakkiyappan, R., Cao, J., Velmurugan, G.: Existence and uniform stability analysis of fractional-order complex-valued neural networks with time delays. IEEE Trans. Neural Netw. Learn. Syst. 26(1), 84-97 (2015)

25. Wang, Z., Shu, H., Liu, Y., Ho, D.W.C., Liu, X.: Robust stability analysis of generalized neural networks with discrete and distributed time delays. Chaos Solitons Fractals 30(4), 886-896 (2006)

26. Li, H.-L., Hu, C., Jiang, H., Teng, Z., Jiang, Y.-L.: Synchronization of fractional-order complex dynamical networks via periodically intermittent pinning control. Chaos Solitons Fractals 103, 357-363 (2017)

27. Yang, X., Li, C., Huang, T., Song, Q., Chen, X.: Quasi-uniform synchronization of fractional-order memristor-based neural networks with delay. Neurocomputing 234, 205-215 (2017)

28. Zhang, H., Ye, R., Liu, S., Cao, J., Alsaedi, A., Li, X.: LMI-based approach to stability analysis for fractional-order neural networks with discrete and distributed delays. Int. J. Syst. Sci. 49(3), 537-545 (2018)

29. Rakkiyappan, R., Velmurugan, G., Cao, J.: Stability analysis of fractional-order complex-valued neural networks with time delays. Chaos Solitons Fractals 78, 297-316 (2015)

30. Simmons, G.F:: Calculus Gems: Brief Lives and Memorable Mathematics. McGraw-Hill, New York (1992)

31. Conway, J.H., Smith, D.A.: On Quaternions and Octonions: Their Geometry, Arithmetic, and Symmetry. AK Peters, Natick (2003)

32. Matsui, N., Isokawa, T., Kusamichi, H., Peper, F., Nishimura, H.: Quaternion neural network with geometrical operators. J. Intell. Fuzzy Syst. 15(3,4), 149-164 (2004)

33. Ujang, B.C., Took, C.C., Mandic, D.P.: Quaternion-valued nonlinear adaptive filtering. IEEE Trans. Neural Netw. 22(8), $1193-1206$ (2011)

34. Chen, X., Song, Q., Li, Z:: Design and analysis of quaternion-valued neural networks for associative memories. IEEE Trans. Syst. Man Cybern. Syst. 48(12), 2305-2314 (2018)

35. Liu, Y., Zhang, D., Lu, J., Cao, J.: Global $\mu$-stability criteria for quaternion-valued neural networks with unbounded time-varying delays. Inf. Sci. 360, 273-288 (2016)

36. Shu, H., Song, Q., Liu, Y., Zhao, Z., Alsaadi, F.E.: Global $\mu$ - stability of quaternion-valued neural networks with non-differentiable time-varying delays. Neurocomputing 247, 202-212 (2017)

37. Zhang, D., Kou, K.I., Liu, Y., Cao, J.: Decomposition approach to the stability of recurrent neural networks with asynchronous time delays in quaternion field. Neural Netw. 94, 55-66 (2017)

38. Liu, Y., Zhang, D., Lou, J., Lu, J., Cao, J.: Stability analysis of quaternion-valued neural networks: decomposition and direct approaches. IEEE Trans. Neural Netw. Learn. Syst. 29(9), 4201-4211 (2018)

39. You, X., Song, Q., Liang, J., Liu, Y., Alsaadi, F.E.: Global $\mu$-stability of quaternion-valued neural networks with mixed time-varying delays. Neurocomputing 290, 12-25 (2018)

40. Chen, X., Li, Z., Song, Q., Hu, J., Tan, Y.: Robust stability analysis of quaternion-valued neural networks with time delays and parameter uncertainties. Neural Netw. 91, 55-65 (2017)

41. Liu, Y., Zhang, D. Lu, J.: Global exponential stability for quaternion-valued recurrent neural networks with time-varying delays. Nonlinear Dyn. 87(1), 553-565 (2017) 
42. Tu, Z., Cao, J., Alsaedi, A., Hayat, T.: Global dissipativity analysis for delayed quaternion-valued neural networks. Neural Netw. 89, 97-104 (2017)

43. Chen, X., Song, Q., Li, Z., Zhao, Z., Liu, Y.: Stability analysis of continuous-time and discrete-time quaternion-valued neural networks with linear threshold neurons. IEEE Trans. Neural Netw. Learn. Syst. 29(7), 2769-2781 (2018)

44. Yang, X., Li, C., Song, Q., Chen, J., Huang, J.: Global Mittag-Leffler stability and synchronization analysis of fractional-order quaternion-valued neural networks with linear threshold neurons. Neural Netw. 105, 88-103 (2018)

45. Chen, X., Song, Q.: State estimation for quaternion-valued neural networks with multiple time delays. IEEE Trans. Syst. Man Cybern. Syst. (2017). https://doi.org/10.1016/j.neunet.2017.04.006

Submit your manuscript to a SpringerOpen ${ }^{\circ}$ journal and benefit from:

- Convenient online submission

- Rigorous peer review

- Open access: articles freely available online

- High visibility within the field

- Retaining the copyright to your article

Submit your next manuscript at $\gg$ springeropen.com 